\title{
Some Considerations about Fuzzy Logic Based Decision Making by Autonomous Intelligent Actor
}

\author{
Alex Tserkovny \\ Applied AI Services, Brookline, USA \\ Email: atserkovny@yahoo.com
}

How to cite this paper: Tserkovny, A. (2022) Some Considerations about Fuzzy Logic Based Decision Making by Autonomous Intelligent Actor. Journal of Software Engineering and Applications, 15, 19-58.

https://doi.org/10.4236/jsea.2022.152002

Received: January 7, 2022

Accepted: February 22, 2022

Published: February 25, 2022

Copyright $\odot 2022$ by author(s) and Scientific Research Publishing Inc. This work is licensed under the Creative Commons Attribution International License (CC BY 4.0).

http://creativecommons.org/licenses/by/4.0/

\begin{abstract}
The article presents an approach toward an implementation of a fuzzy logic-based decision-making process by Autonomous Intelligent Actor (AIA) (C) A. Tserkovny), when an input information is defined for its "strategic targeting" by a human operator in terms of a fuzzy incident geometry, whereas its "tactical" behavior (a navigation in space) is directed by fuzzy conditional inference rules. For implementing both elements of AIA decision-making a fuzzy logic [1] for formal geometric reasoning with extended objects is used. This fuzzy logic based fuzzification of axioms of an incidence geometry and a predicate apparatus [2] for AIA space orientation are also presented. The approach, offered in the article, extends predicates of a counter positioning of two objects and their mutual navigation into their fuzzy counterparts. The latter allows AIA to make certain "tactical" decisions.
\end{abstract}

\section{Keywords}

Fuzzy Logic, Implication, Conjunction, Disjunction, Fuzzy Predicate, Degree of Indiscernibility, Discernibility Measure, Extended Lines Sameness, AIA Orientation Principles

\section{Introduction}

The article introduces the notion of an Autonomous Intelligent Actor (AIA), which, in general terms, represents an entity, which would be able to make an independent decision about strategic and tactical behavior, given an ultimate goal, defined by a human operator. For this purpose, we have proposed to use both fuzzy incident geometry paradigm for AIA strategic planning and fuzzy conditional inference rules for its local orientation (tactical behavior). The article 
presents a fuzzy incident geometry (๑ A. Tserkovny), based on a fuzzy logic [1] (๔ A. Tserkovny). For a consistency's sake we use the same fuzzy logic for an AIA orientation, once the strategic positioning is achieved.

\section{Axiomatic Geometry and Extended Objects for AIA Strategic Targeting}

In this chapter we consider AIA Strategic Targeting Apparatus to be defined by not traditional primitives of Euclidean Geometry, such as points and lines, but by their extended in space counterparts. In other words, we are reinstating Euclidean geometry, including the concepts of crisp points and lines, by using different set of geometric primitives. One of our goals is to expand traditional axiomatization of Euclidean geometry by applying fuzzification technique.

Similarly, to [3] we will present a set of incident geometry axiom, which validates the conduct of points and lines in space.

I1) Every two separate points $p$ and $q$ could be linked by at least one line 1, which is incident both.

D) Such a line is unique.

B) Every line is incident with at least two points.

I4) At least three points exist that are not incident with the same line.

Now we formulate some entities of geometry by applying so-called construction operators sequentially. An instance of a construction operator is

\section{Connect: point $\times$ point $\rightarrow$ line.}

It means these input two points are connected by the line through them. In accordance with axiom $I 2$ Connect to is well-defined mathematical function, because resulting line is unique and always exists. Let us show that couple more examples of geometric construction operators of 2D incidence geometry are

\section{Intersect: line $\times$ line $\rightarrow$ point, \\ Parallel through point. line $\times$ point $\rightarrow$ line}

Note that the set of axioms of incidence geometry is just a subset of the axioms of Euclidean geometry.

\section{Fuzzification of Incidence Geometry}

\subsection{Fuzzy Logic in Use}

Now we present some basic operations in a fuzzy logic [1] we will use for all purposes of an article. We define the truth values of logical antecedent $A$ and consequent $B$ as $T(A)=a$ and $T(B)=b$ respectively. Then relevant set of proposed fuzzy logic operators is shown in Table 1 . To get the truth values of these definitions we use well known logical properties such as $A \rightarrow b=\neg a \vee b$; $a \wedge b=\neg(\neg a \vee \neg b)$ and alike. In other words we are considering a many-valued system, characterized by the set of base union $(U)$ and intersection $(\cap)$ operations with relevant complement, defined as $T(\neg A)=1-T(A)$. In addition, the operators $\downarrow$ and $\uparrow$ are expressed as negations of the $U$ and $\cap$ correspondingly. 
From practical point of view and for illustration purposes only the real interval $\mathfrak{R}=[0,1]$ would be presented by the set of 11 values, i.e., we are considering the following set $V_{11}=\{0,0.1,0.2, \cdots, 0.9,1\}$, which we shall use as a universe of discourse in all our future exercises. Table 2 shows the operation implication

Table 1. The logical operations of a fuzzy logic in use.

\begin{tabular}{|c|c|c|}
\hline Name & Designation & Value \\
\hline Tautology & $A^{\mathrm{I}}$ & 1 \\
\hline Controversy & $A^{\circ}$ & 0 \\
\hline Negation & $\neg A$ & $1-A$ \\
\hline Disjunction & $A \vee B$ & $\left\{\begin{array}{l}a \cdot b, a+b<1 \\
1, a+b \geq 1\end{array}\right.$ \\
\hline Conjunction & $A \wedge B$ & $\left\{\begin{array}{l}a \cdot b, a+b>1 \\
0, a+b \leq 1\end{array}\right.$ \\
\hline Implication & $A \rightarrow B$ & $\left\{\begin{array}{l}(1-a) \cdot b, a>b \\
1, a \leq b\end{array}\right.$ \\
\hline Equivalence & $A \leftrightarrow B$ & $\left\{\begin{array}{l}(1-a) \cdot b, a<b \\
1, a=b \\
(1-b) \cdot a, b<a\end{array}\right.$ \\
\hline Pierce Arrow & $A \downarrow B$ & $\left\{\begin{array}{l}(1-a) \cdot(1-b), a+b<1 \\
0, a+b \geq 1\end{array}\right.$ \\
\hline Shaffer Stroke & $A \uparrow B$ & $\left\{\begin{array}{l}(1-a) \cdot(1-b), a+b>1 \\
1, a+b \leq 1\end{array}\right.$ \\
\hline
\end{tabular}

Table 2. The operation implication $I(a, b)$ in fuzzy logic in use.

\begin{tabular}{cccccccccccc}
\hline$a \rightarrow b$ & 0 & 0.1 & 0.2 & 0.3 & 0.4 & 0.5 & 0.6 & 0.7 & 0.8 & 0.9 & 1 \\
\hline 0 & 1 & 1 & 1 & 1 & 1 & 1 & 1 & 1 & 1 & 1 & 1 \\
0.1 & 0 & 1 & 1 & 1 & 1 & 1 & 1 & 1 & 1 & 1 & 1 \\
0.2 & 0 & 0.08 & 1 & 1 & 1 & 1 & 1 & 1 & 1 & 1 & 1 \\
0.3 & 0 & 0.07 & 0.14 & 1 & 1 & 1 & 1 & 1 & 1 & 1 & 1 \\
0.4 & 0 & 0.06 & 0.12 & 0.18 & 1 & 1 & 1 & 1 & 1 & 1 & 1 \\
0.5 & 0 & 0.05 & 0.1 & 0.015 & 0.2 & 1 & 1 & 1 & 1 & 1 & 1 \\
0.6 & 0 & 0.04 & 0.08 & 0.12 & 0.16 & 0.2 & 1 & 1 & 1 & 1 & 1 \\
0.7 & 0 & 0.03 & 0.06 & 0.09 & 0.12 & 0.15 & 0.18 & 1 & 1 & 1 & 1 \\
0.8 & 0 & 0.02 & 0.04 & 0.06 & 0.08 & 0.1 & 0.12 & 0.14 & 1 & 1 & 1 \\
0.9 & 0 & 0.01 & 0.02 & 0.03 & 0.04 & 0.05 & 0.06 & 0.07 & 0.08 & 1 & 1 \\
1 & 0 & 0 & 0 & 0 & 0 & 0 & 0 & 0 & 0 & 0 & 1 \\
\hline
\end{tabular}


in fuzzy logic in use, which unique feature is the following

$$
F_{1}(a, b)=(1-a) \cdot b, a>b
$$

From where we have

$$
I(a, b)=\left\{\begin{array}{l}
F_{1}(a, b), a>b ; \\
1, a \leq b,
\end{array}=\left\{\begin{array}{l}
(1-a) \cdot b, a>b, \\
1, a \leq b
\end{array}\right.\right.
$$

\subsection{Geometric Primitives as Fuzzy Predicates}

Let us denote some incidence geometry predicates as $p(a)$ (“ $a$ is a point”), $l(a)$ (" $a$ is a line"), and inc $(a, b)$ (" $a$ and $b$ are incident"). Traditionally predicates are interpreted by crisp relations. The predicate expressing equality can be denotes by eq( $a$, $b$ ) (" $a$ and $b$ are equal"). For example, eq: $N \times N \rightarrow\{0,1\}$ is a switch function between every pair of equal to every pair of distinct objects from the set $N$. Both predicates $p($.$) and l($.$) , with only one symbol input argument are unary, whereas$ binary predicates, like inc(...) and $e q(. .$.$) , accept pairs of symbols as an input. In this$ work by applying fuzzy predicate logic, we are re-interpreting all types of crisp relations predicates by their fuzzy counterparts. For instance, a binary fuzzy relation eq is defined as function $e q: N \times N \rightarrow[0,1]$, assigning a real number $\lambda \in[0,1]$ to every pair of objects from $N$. In other words, every two objects of $N$ are equal to some degree. The degree of equality of two objects $a$ and $b$ may be 1 or 0 as in the crisp case, but can as well be 0.9 , expressing that $a$ and $b$ are almost equal.

Note that point-predicate $p($.$) for Cartesian point does not change when the$ point is rotated, i.e. rotation-invariance could be a main characteristic of "point likeness" with respect to geometric operations. In other words "point likeness" should be kept in a relevant fuzzy predicate expressing the extended subsets of $R^{1}$ Let us define

$$
\begin{aligned}
& \theta_{\text {min }}(A)=\min _{t}\left|\operatorname{ch}(A) \cap\left\{c(A)+t \cdot R_{\alpha} \cdot(0,1)^{T} \mid t \in \mathfrak{R}\right\}\right| \\
& \theta_{\max }(A)=\max _{t}\left|\operatorname{ch}(A) \cap\left\{c(A)+t \cdot R_{\alpha} \cdot(0,1)^{T} \mid t \in \mathfrak{R}\right\}\right|
\end{aligned}
$$

as the minimal and maximal diameter of the convex hull $\operatorname{ch}(A)$ of $A \subseteq \operatorname{Dom}$, respectively. The convex hull in certain way normalizes (eliminates irregularities) the sets A and B. $c(A)$ denotes the centroid of $c h(A)$, and $R \alpha$ denotes the rotation matrix by angle $\alpha$ (Figure 1 (a)) [3].

Let's describe the fuzzy point-predicate $p($.$) , given the fact that A$ set is bounded and both $c h(A)$ and $c(A)$ exist, by

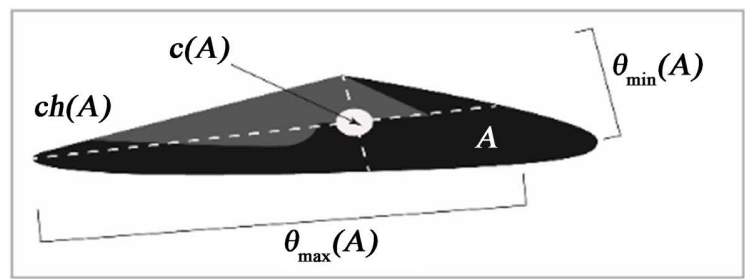

(a)

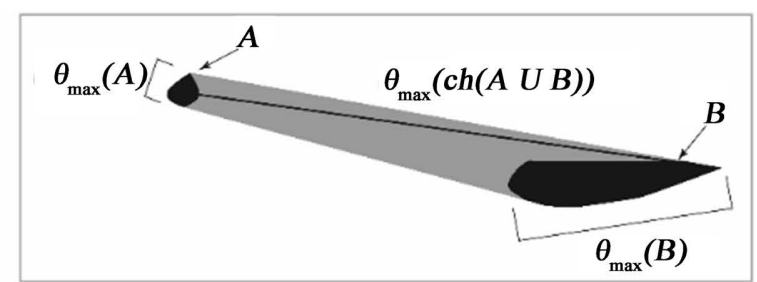

(b)

Figure 1. (a) Cartesian point A minimal and maximal diameters. Note: (b) A and B point's distinctness. 


$$
p(A)=\theta_{\min }(A) / \theta_{\max }(A) .
$$

If we express the degree to which the convex hull of a Cartesian point set $A$ is rotation-invariant as $A \subseteq D o m p($.) and if $p(A)=1$, then $c h(A)$ is perfectly rotation invariant and it is a disc. And since $A$ is assumed to be two-dimensional, then inequality $\theta_{\max }(A) \neq 0$ always holds. In addition to $p($.$) , the fuzzy$ line-predicate is defined as a compliment to fuzzy-point one

$$
l(A)=1-p(A)
$$

To define the degree to which a Cartesian point set $A \subseteq D o m$ is sensitive to rotation and since we only regard convex hulls, then a fuzzy version of the incidence-predicate inc(...) would be a binary fuzzy relation between Cartesian point sets $A, B \subseteq D o m$ :

$$
\operatorname{inc}(A, B)=\max (|\operatorname{ch}(A) \cap \operatorname{ch}(B)| /|\operatorname{ch}(A)|,|\operatorname{ch}(A) \cap \operatorname{ch}(B)| /|\operatorname{ch}(B)|)
$$

Here in (2.6) we select the greater one of two convex hulls of $A$ and $B$ and this fuzzy relation measures the relative overlaps of them. Here $A$ and $B$ are considered as an incident to degree one, if $|\operatorname{ch}(A)|$ denotes the area occupied by $\operatorname{ch}(A)$. The greater inc $(A, B)$, "the more incident" are $A$ and $B$ : If $A \subseteq B$ or $B \subseteq A$, then $\operatorname{inc}(A, B)=1$.

Contrariwise to inc(...), we define a graduated equality predicate $e q(. .$.$) be-$ tween the bounded Cartesian point sets $A, B \subseteq$ Dom as follows:

$$
e q(A, B)=\min (|\operatorname{ch}(A) \cap \operatorname{ch}(B)| /|\operatorname{ch}(A)|,|\operatorname{ch}(A) \cap \operatorname{ch}(B)| /|\operatorname{ch}(B)|)
$$

where $e q(A, B)$ measures the minimal relative overlap of $A$ and $B$, whereas the negation $\neg e q(A, B)=1-e q(A, B)$ measures the degrees to which the two-point sets do not overlap: if $e q(A, B) \approx 0$, then $A$ and $B$ are "almost disjoint".

Then we can define the following measure of "distinctness of points" $d p($.) of two extended objects from Figure 1(b) as

$$
d p(A, B)=\max \left(0,1-\max \left(\theta_{\max }(A), \theta_{\max }(B)\right) / \theta_{\max }(\operatorname{ch}(A \cup B))\right)
$$

It is apparent, that the greater $d p(A, B)$, the more $A$ and $B$ behave like distinct Cartesian points with respect to connection. Indeed, for Cartesian points $a$ and $b$, we would have $d p(A, B)=1$. If the distance between the Cartesian point sets $A$ and $B$ is infinitely big, then $d p(A, B)=1$ as well. If $\max \left(\theta_{\max }(A), \theta_{\text {max }}(B)\right)>\theta_{\text {max }}(\operatorname{ch}(A \cup B))$ then $d p(A, B)=0$.

\subsection{Formalization of Fuzzy Predicates}

To formalize fuzzy predicates, defined in subchapter 2.2 both implication $\rightarrow$ and conjunction operators are defined as in Table 1:

$$
\begin{gathered}
A \wedge B=\left\{\begin{array}{l}
a \cdot b, a+b>1, \\
0, a+b \leq 1
\end{array}\right. \\
A \rightarrow B=\left\{\begin{array}{l}
(1-a) \cdot b, a>b, \\
1, a \leq b
\end{array}\right.
\end{gathered}
$$


In our further discussions we will also use the disjunction operator from the same table.

$$
A \vee B=\left\{\begin{array}{l}
a \cdot b, a+b<1, \\
1, a+b \geq 1
\end{array}\right.
$$

Now let us re-define the set of fuzzy predicates (2.6)-(2.8), using proposed fuzzy logic's operators.

PROPOSITION 1.

If fuzzy predicate ind (...) is defined as in (2.6) and conjunction operator is defined as in (2.9), then

$$
\operatorname{inc}(A, B)=\left\{\begin{array}{l}
\max (a, b), a+b>1, \\
0, a+b \leq 1
\end{array}\right.
$$

Proof: Let's present (2.6) as follows:

$$
\frac{|\operatorname{ch}(A) \cap \operatorname{ch}(B)|}{|\operatorname{ch}(A)|}=\frac{A \cdot B}{A}=B \text { and } \frac{|\operatorname{ch}(A) \cap \operatorname{ch}(B)|}{|\operatorname{ch}(B)|}=\frac{A \cdot B}{B}=A
$$

Therefore from (2.6) and (2.13) we are getting (2.12). (Q.E.D.).

\section{PROPOSITION 1.}

If fuzzy predicate $e q(\ldots)$ is defined as in (2.7) and disjunction operator is defined as in (2.11), then

$$
e q(A, B)=\left\{\begin{array}{l}
\min (a, b), a+b<1, \\
1, a+b \geq 1
\end{array}\right.
$$

Proof:

From (2.7) and (2.13) we are getting (2.14). (Q.E.D.).

\section{COROLLARY 1.}

If fuzzy predicate $e q(A, B)$ is defined as (2.14), then the following type of transitivity is taking place

$$
e q(a, c) \rightarrow e q(a, b) \wedge e q(b, c)
$$

where $A, B, C \subseteq D o m$, and Dom is partially ordered space, i.e., either $A \subseteq B \subseteq C$ or wise versa. (Note: both conjunction and implication operations are defined in Table 1).

Proof:

From (2.14) we have

$$
\begin{aligned}
& e q(A, C)=\left\{\begin{array}{l}
\min (a, c), a+c<1, \\
1, a+c \geq 1
\end{array},\right. \\
& e q(A, B)=\left\{\begin{array}{l}
\min (a, b), a+b<1, \\
1, a+b \geq 1
\end{array}\right. \\
& e q(B, C)=\left\{\begin{array}{l}
\min (b, c), b+c<1, \\
1, b+c \geq 1
\end{array}\right.
\end{aligned}
$$

Let $a<b<c \leq 1$ and $a+c<1, a+b<1, b+c<1$ from (2.16) we have 


$$
\begin{aligned}
& e q(a, c)=\min (a, c), \\
& e q(a, b)=\min (a, b), \\
& e q(b, c)=\min (b, c) .
\end{aligned}
$$

From (2.17), given $a<b<c \leq 1$, we are getting

$$
\begin{aligned}
& e q(a, c)=a, \\
& e q(a, b)=a, \\
& e q(b, c)=b .
\end{aligned}
$$

Given (2.9) and (2.18) we have $a<a \cdot b$ and therefore

$$
e q(a, c) \rightarrow e q(a, b) \wedge e q(b, c)
$$

From (2.17), given $a>b>c \leq 1$, we are getting

$$
\begin{aligned}
& e q(a, c)=c, \\
& e q(a, b)=b, \\
& e q(b, c)=c .
\end{aligned}
$$

Given (2.9) and (2.19) we have $c<b \cdot c$ and therefore

$$
e q(a, c) \rightarrow e q(a, b) \wedge e q(b, c) .(\text { Q.E.D. }) .
$$

\section{PROPOSITION 2.}

If fuzzy predicate $d p(\ldots)$ is defined as in (2.8) and disjunction operator is defined as in (2.11), then

$$
d p(A, B)=\left\{\begin{array}{l}
1-a, a+b \geq 1 \& a \geq b, \\
1-b, a+b \geq 1 \& a<b, \\
0, a+b<1
\end{array}\right.
$$

Proof:

From (2.8) we get the following:

$$
d p(A, B)=\max \left\{0,1-\frac{\max (A, B)}{A \cup B}\right\}
$$

For the case, when $a+b<1$, and given (2.11)

$$
d p(A, B)=\max \left\{0,1-\frac{\max (a, b)}{a \cdot b}\right\}=\left\{\begin{array}{l}
\max \left\{0,1-\frac{1}{b}\right\}, a>b, \\
\max \left\{0,1-\frac{1}{a}\right\}, a<b .
\end{array}\right.
$$

Since $1-\frac{1}{b}<0$ and $1-\frac{1}{a}<0$, given $a, b \in[0,1]$ and from (2.21) we are getting

$$
d p(A, B) \equiv 0 .
$$

For the case, when $a+b \geq 1$, and given (2.11)

$d p(A, B)=\max \left\{0,1-\frac{\max (a, b)}{a \cdot b}\right\}=\left\{\begin{array}{l}\max \{0,1-a\}, a>b, \\ \max \{0,1-b\}, a<b .\end{array}=\left\{\begin{array}{l}1-a, a>b, \\ 1-b, a<b .\end{array}\right.\right.$

From (2.22) and (2.23) we are getting (2.20) (Q.E.D.). 


\subsection{Fuzzy Axiomatization of Incidence Geometry}

By using the fuzzy predicates formalized in subchapter 2.3, we propose the set of axioms as fuzzy version of incidence geometry in the language of a fuzzy logic [1] as follows:

$$
\begin{array}{ll}
\text { I1': } & d p(a, b) \rightarrow \sup _{c}[l(c) \wedge \operatorname{inc}(a, c) \wedge \operatorname{inc}(b, c)] \\
& d p(a, b) \rightarrow\left[l ( c ) \rightarrow \left[\operatorname { i n c } ( a , c ) \rightarrow \left[\operatorname { i n c } ( b , c ) \rightarrow l ( c ^ { \prime } ) \rightarrow \left[\operatorname{inc}\left(a, c^{\prime}\right)\right.\right.\right.\right. \\
\text { I2': } \quad & \left.\left.\left.\left.\rightarrow\left[\operatorname{inc}\left(b, c^{\prime}\right) \rightarrow e q\left(c, c^{\prime}\right)\right]\right]\right]\right]\right] \\
\text { I3': } & l(c) \rightarrow \sup _{a, b}\{p(a) \wedge p(b) \wedge \neg e q(a, b) \wedge \operatorname{inc}(a, c) \wedge \operatorname{inc}(b, c)\} \\
\text { I4': } \sup _{a, b, c, d}[p(a) \wedge p(b) \wedge p(c) \wedge l(d) \rightarrow \neg(\operatorname{inc}(a, d) \wedge \operatorname{inc}(b, d) \wedge \operatorname{inc}(c, d))]
\end{array}
$$

In axioms I1'-I4' we also use a set of operations (2.9)-(2.11).

\section{PROPOSITION 3.}

If fuzzy predicates $d p(\ldots)$ and inc(...) are defined like (2.20) and (2.12) respectively, then axiom $I 1$ 'is fulfilled for the set of logical operators from a fuzzy logic [1]. (For every two distinct point $a$ and $b$, at least one line 1 exists that is incident with $a$ and $b$.)

Proof:

From (2.15)

$$
\begin{aligned}
& \operatorname{inc}(A, C)=\left\{\begin{array}{l}
\max (a, c), a+c>1, \\
0, a+c \leq 1
\end{array}\right. \\
& \operatorname{inc}(B, C)=\left\{\begin{array}{l}
\max (b, c), b+c>1, \\
0, b+c \leq 1
\end{array}\right.
\end{aligned}
$$

Given (2.9) we are getting

$$
\operatorname{inc}(A, C) \wedge \operatorname{inc}(B, C)=\left\{\begin{array}{l}
\operatorname{inc}(A, C) \cdot \operatorname{inc}(B, C), \operatorname{inc}(A, C)+\operatorname{inc}(B, C)>1, \\
0, \operatorname{inc}(A, C)+\operatorname{inc}(B, C) \leq 1
\end{array}\right.
$$

Given (2.24) and (2.25)

$$
\operatorname{inc}(A, C) \wedge \operatorname{inc}(B, C)=\left\{\begin{array}{l}
\max (A, C) \cdot \max (B, C), \max (A, C)+\max (B, C)>1, \\
0, \max (A, C)+\max (B, C) \leq 1
\end{array}\right.
$$

But

$$
\begin{aligned}
& \sup _{c}[l(c) \wedge \operatorname{inc}(a, c) \wedge \operatorname{inc}(b, c)] \\
& =\sup _{c}\left[l(c) \wedge\left\{\begin{array}{l}
C^{2}, 2 \cdot C>1, \\
0,2 \cdot C \leq 1
\end{array}\right]=\sup _{c}\left[l(c) \wedge\left\{\begin{array}{l}
C^{2}, C>0.5, \\
0, C \leq 0.5
\end{array}\right]\right.\right.
\end{aligned}
$$

In (2.27) we have the following

$$
\left\{\begin{array}{l}
C^{2}, C>0.5, \\
0, C \leq 0.5
\end{array} \in(0.25,1]\right.
$$

From (2.28) we are getting 


$$
\sup _{c}\left[l(c) \wedge\left\{\begin{array}{l}
C^{2}, C>0.5, \\
0, C \leq 0.5
\end{array}\right] \equiv 1\right.
$$

From (2.20) we always have $d p(A, B)<1$, therefore

$$
d p(a, b) \rightarrow \sup _{c}[l(c) \wedge \operatorname{inc}(a, c) \wedge \operatorname{inc}(b, c)] \text { (Q.E.D.). }
$$

\section{PROPOSITION 4.}

If fuzzy predicates $d p(\ldots), e q(\ldots)$ and inc (..) are defined like (2.35), (2.16) and (2.15) respectively, then axiom I2' is fulfilled for the set of logical operators from a fuzzy logic [1]. (For every two distinct point $a$ and $b$, at least one line $I$ exists that is incident with $a$ and $b$ and such a line is unique)

Proof:

Let's take a look at the following implication:

$$
\operatorname{inc}\left(b, c^{\prime}\right) \rightarrow e q\left(c, c^{\prime}\right)
$$

But from (2.14) we have

$$
e q\left(C, C^{\prime}\right)=\left\{\begin{array}{l}
\min \left(c, c^{\prime}\right), c+c^{\prime}<1, \\
1, c+c^{\prime} \geq 1
\end{array}\right.
$$

And from (2.15)

$$
\operatorname{inc}\left(B, C^{\prime}\right)=\left\{\begin{array}{l}
\max \left(b, c^{\prime}\right), b+c^{\prime}>1, \\
0, b+c^{\prime} \leq 1
\end{array}\right.
$$

From (2.31) and (2.32) we see, that $\operatorname{inc}\left(B, C^{\prime}\right) \leq e q\left(C, C^{\prime}\right)$, which means that

$$
\operatorname{inc}\left(b, c^{\prime}\right) \rightarrow e q\left(c, c^{\prime}\right) \equiv 1 \text {, }
$$

Therefore, the following is also true

$$
\operatorname{inc}\left(a, c^{\prime}\right) \rightarrow\left[\operatorname{inc}\left(b, c^{\prime}\right) \rightarrow e q\left(c, c^{\prime}\right)\right] \equiv 1
$$

Now let's look at the following implication $\operatorname{inc}(b, c) \rightarrow l\left(c^{\prime}\right)$. Since $\operatorname{inc}(b, c) \geq l\left(c^{\prime}\right)$, we are getting $\operatorname{inc}(b, c) \rightarrow l\left(c^{\prime}\right) \equiv 0$. Considering (2.33) we have the following

$$
\operatorname{inc}(b, c) \rightarrow l\left(c^{\prime}\right) \rightarrow\left[\operatorname{inc}\left(a, c^{\prime}\right) \rightarrow\left[\operatorname{inc}\left(b, c^{\prime}\right) \rightarrow e q\left(c, c^{\prime}\right)\right]\right] \equiv 1
$$

Since from (2.12) $\operatorname{inc}(a, c) \leq 1$, then with taking into account (2.34) we've gotten the following:

$\operatorname{inc}(a, c) \rightarrow\left[\operatorname{inc}(b, c) \rightarrow l\left(c^{\prime}\right) \rightarrow\left[\operatorname{inc}\left(a, c^{\prime}\right) \rightarrow\left[\operatorname{inc}\left(b, c^{\prime}\right) \rightarrow e q\left(c, c^{\prime}\right)\right]\right]\right] \equiv 1$

Since $l(c) \leq 1$, from $(2.35)$ we are getting:

$$
\begin{aligned}
& l(c) \rightarrow\left[\operatorname { i n c } ( a , c ) \rightarrow \left[\operatorname { i n c } ( b , c ) \rightarrow l ( c ^ { \prime } ) \rightarrow \left[\operatorname{inc}\left(a, c^{\prime}\right)\right.\right.\right. \\
& \left.\left.\left.\rightarrow\left[\operatorname{inc}\left(b, c^{\prime}\right) \rightarrow e q\left(c, c^{\prime}\right)\right]\right]\right]\right] \equiv 1
\end{aligned}
$$

Finally, because $d p(a, b) \leq 1$ we have

$$
\begin{aligned}
d p(a, b) \leq & \left\{l ( c ) \rightarrow \left[\operatorname { i n c } ( a , c ) \rightarrow \left[\operatorname { i n c } ( b , c ) \rightarrow l ( c ^ { \prime } ) \rightarrow \left[\operatorname{inc}\left(a, c^{\prime}\right)\right.\right.\right.\right. \\
& \left.\left.\left.\left.\rightarrow\left[\operatorname{inc}\left(b, c^{\prime}\right) \rightarrow e q\left(c, c^{\prime}\right)\right]\right]\right]\right]\right\}
\end{aligned}
$$


(Q.E.D.).

\section{PROPOSITION 6.}

If fuzzy predicates $e q(\ldots)$ and inc(...) are defined like (2.14) and (2.12) respectively, then axiom I3' is fulfilled for the set of logical operators from a fuzzy logic [1]. (Every line is incident with at least two points.)

Proof:

It was already shown in (2.28) that $\operatorname{inc}(a, c) \wedge \operatorname{inc}(b, c) \in(0.25,1]$

And from (2.14) we have

$$
e q(A, B)=\left\{\begin{array}{l}
\min (a, b), a+b<1, \\
1, a+b \geq 1
\end{array}\right.
$$

The negation $\neg e q(A, B)$ will be

$$
\neg e q(A, B)=\left\{\begin{array}{l}
\max (a, b), a+b<1, \\
0, a+b \geq 1
\end{array}\right.
$$

Given (2.36) and (2.9) we get

$$
\neg e q(A, B) \wedge 1=\left\{\begin{array}{l}
\max (a, b), \max (a, b)+1>0, \\
0, \text { otherwise }
\end{array}=\max (a, b)\right.
$$

But

$\sup _{a, b}\{p(a) \wedge p(b) \wedge \neg e q(a, b) \wedge \operatorname{inc}(a, c) \wedge \operatorname{inc}(b, c)\}=1 \wedge \max (a, b) \wedge a \cdot b=1$.

And given, that $l(c)<1$ we are getting

$$
l(c) \rightarrow \sup _{a, b}\{p(a) \wedge p(b) \wedge \neg e q(a, b) \wedge i n c(a, c) \wedge i n c(b, c)\} \equiv 1 \text { (Q.E.D.). }
$$

\section{PROPOSITION 7.}

If fuzzy predicate inc(...) is defined like (2.15), then axiom I4' is fulfilled for the set of logical operators from a fuzzy logic [1]. (At least three points exist that are not incident with the same line.)

Proof:

From (2.12) we have

$$
\begin{aligned}
\operatorname{inc}(A, D) & =\left\{\begin{array}{l}
\max (a, d), a+d>1, \\
0, a+d \leq 1
\end{array}\right. \\
\operatorname{inc}(B, D) & =\left\{\begin{array}{l}
\max (b, d), b+d>1, \\
0, b+d \leq 1
\end{array}\right. \\
\operatorname{inc}(C, D) & =\left\{\begin{array}{l}
\max (c, d), c+d>1, \\
0, c+d \leq 1
\end{array}\right.
\end{aligned}
$$

But from (2.36) which we have

$(\operatorname{inc}(a, d) \wedge \operatorname{inc}(b, d) \wedge \operatorname{inc}(c, d))=(\max (a, d) \wedge \max (b, d) \wedge \max (c, d))$

From (2.38)

$$
\neg(\operatorname{inc}(a, d) \wedge \operatorname{inc}(b, d) \wedge \operatorname{inc}(c, d))=\neg(\max (a, d) \wedge \max (b, d) \wedge \max (c, d))
$$


From (2.39)

$\neg(\max (a, d) \wedge \max (b, d) \wedge \max (c, d))=(\min (a, d) \vee \min (b, d) \vee \min (c, d))$ or $(\operatorname{inc}(a, d) \wedge \operatorname{inc}(b, d) \wedge \operatorname{inc}(c, d))=1 \wedge \operatorname{inc}(c, d) \equiv 1$, from where we have $\neg(\operatorname{inc}(a, d) \wedge \operatorname{inc}(b, d) \wedge \operatorname{inc}(c, d)) \equiv 0$. Since $l(d) \equiv 0 \mid d=1$ we are getting $l(d)=\neg(\operatorname{inc}(a, d) \wedge \operatorname{inc}(b, d) \wedge \operatorname{inc}(c, d))$, which could be interpreted like $l(d) \rightarrow \neg(\operatorname{inc}(a, d) \wedge \operatorname{inc}(b, d) \wedge \operatorname{inc}(c, d))=1$, from which we finally get the following $\sup _{a, b, c, d}[p(a) \wedge p(b) \wedge p(c) \wedge 1] \equiv 1$ (Q.E.D.).

\subsection{Equality of Extended Lines Is Graduated}

In [3] it was shown that the location of the extended points creates a constraint on the location of an incident extended line. It was also mentioned, that in traditional geometry this location constraint fixes the position of the line uniquely. And therefore, in case points and lines are allowed to have extension this is not the case. Consequently, Euclid's First postulate does not apply: Figure 2 shows that if two distinct extended points $P$ and $Q$ are incident (i.e., overlap) with two extended lines $L$ and $M$, then $L$ and $M$ are not necessarily equal.

Yet, in most cases, $L$ and $M$ are "closer together", i.e., "more equal" than arbitrary extended lines that have only one or no extended point in common. The further $P$ and $Q$ move apart from each other, the more similar $L$ and $M$ become. One way to model this fact is to allow degrees of equality for extended lines. In other words, the equality relation is graduated: It allows not only for Boolean values, but for values in the whole interval $[0,1]$.

\subsection{Incidence of Extended Points and Lines}

As it was demonstrated in [3], there is a reasonable assumption to classify an extended point and an extended line as incident, if their extended representations in the underlying metric space overlap. We do this by modelling incidence by the subset relation:

Definition 1: For an extended point $A$, and an extended line $L$ we define the incidence relation by

$$
R_{\text {inc }}(A, L):=(A \subseteq L) \in\{0,1\},
$$

where the subset relation $\subseteq$ refers to $A$ and $L$ as subsets of the underlying metric space. The extended incidence relation (2.40) is a Boolean relation, assuming either the truth value 1 (true) or the truth value 0 (false). It is well known that since a Boolean relation is a special case of a graduated relation, i.e., since $\{0,1\} \subset[0,1]$, we will be able to use relation $(2.40)$ as part of fuzzified Euclid's

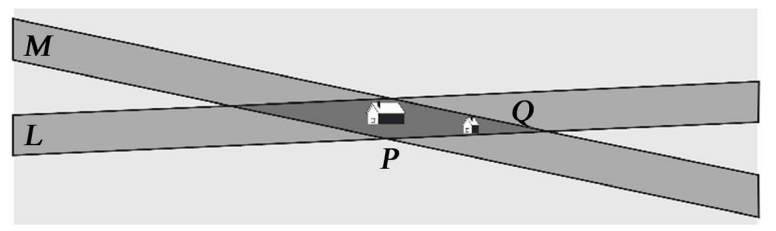

Figure 2. Two extended points do not uniquely determine the location of an incident extended line. 
first postulate later on.

\subsection{Equality of Extended Points and Lines}

As stated in previous chapters, equality of extended points, and equality of extended lines is a matter of degree. Geometric reasoning with extended points and extended lines relies heavily on the metric structure of the underlying coordinate space. Consequently, it is reasonable to model graduated equality as inverse to distance.

\subsubsection{Metric Distance}

In [3] was mentioned that a pseudo metric distance, or pseudo metric, is a map $d: M^{2} \rightarrow \Re^{+}$from domain $M$ into the positive real numbers (including zero), which is minimal, symmetric, and satisfies the triangle inequality:

$$
\forall a, b \in[0,1] \Rightarrow\left\{\begin{array}{l}
d(a, a)=0 \\
d(a, b)=d(b, a) \\
d(a, b)+d(b, c) \geq d(a, c) .
\end{array}\right.
$$

$d$ is called a metric, if additionally holds:

$$
d(a, b)=0 \Leftrightarrow a=b,
$$

Well known examples of metric distances are the Euclidean distance, or the Manhattan distance. Another example is the elliptic metric for the projective plane defined in (2.42) [3]. The "upside-down-version" of a pseudo metric distance is a fuzzy equivalence relation w.r.t. in proposed t-norm fuzzy logic. We will use this particular fuzzy logic to formalize Euclid's first postulate for extended primitives in chapter 4 . The reason for choosing a proposed fuzzy logic is its strong connection to metric distance.

\subsubsection{Fuzzy Equivalence Relations}

As mentioned above, the "upside-down-version" of a pseudo metric distance is a fuzzy equivalence relation w.r.t. the proposed t-norm $\wedge^{\wedge}$. A fuzzy equivalence relation is a fuzzy relation $e: M^{2} \rightarrow[0,1]$ on a domain $M$, which is reflexive, symmetric and $\wedge$-transitive:

$$
\forall a, b \in[0,1] \Rightarrow\left\{\begin{array}{l}
e(a, b)=1 \\
e(a, b)=e(b, a) \\
e(a, b) \wedge e(b, c) \leq e(a, c) .
\end{array}\right.
$$

\section{PROPOSITION 9.}

If Fuzzy Equivalence Relation is defined (Table 1) as the following

$$
e(a, b)=A \leftrightarrow B=\left\{\begin{array}{l}
(1-a) \cdot b, a<b, \\
1, a=b \\
(1-b) \cdot a, b<a,
\end{array}\right.
$$

then conditions (2.43) are satisfied.

Proof: 
1) Reflexivity: $e(a, a)=1$ comes from (2.44) because $a \equiv a$.

2) Symmetricity: $e(a, b)=e(b, a)$.

$$
e(a, b)=\left\{\begin{array}{l}
(1-a) \cdot b, a<b, \\
1, a=b \quad, \text { but } e(b, a)=\left\{\begin{array}{l}
(1-b) \cdot a, b<a, \\
1, a=b \\
(1-b) \cdot a, b<a,
\end{array},\right. \text { therefore } \\
e(a, b) \equiv e(b, a) \text { (Q.E.D.). }
\end{array}\right.
$$

3) Transitivity: $e(a, b) \wedge e(b, c) \leq e(a, c) \mid \forall a, b, c \in L[0,1]$-lattice.

$$
\begin{aligned}
& \text { From (2.52) let } F_{1}(a, c)=e(a, c)=\left\{\begin{array}{l}
(1-a) \cdot c, a<c, \\
1, a=c \\
(1-c) \cdot a, c<a,
\end{array}\right. \\
& \text { and } e(b, c)=\left\{\begin{array}{l}
(1-b) \cdot c, b<c, \\
1, b=c \\
(1-c) \cdot b, c<b,
\end{array},\right. \text { then } \\
& F_{2}(a, c)=e(a, b) \wedge e(b, c)=\left\{\begin{array}{l}
e(a, b) \cdot e(b, c), e(a, b)+e(b, c)>1 \\
0, e(a, b)+e(b, c) \leq 1
\end{array}\right.
\end{aligned}
$$

But

$$
e(a, b) \cdot e(b, c)=\left\{\begin{array}{l}
(1-a) \cdot b \cdot(1-b) \cdot c, a>b>c, \\
1, a=b=c, \\
a \cdot b \cdot(1-b) \cdot(1-c), a<b<c
\end{array}\right.
$$

But since in (2.47) $\forall b \in[0,1] \Rightarrow f(b)=b \cdot(1-b) \in[0,0.25]$ and therefore from (2.45) and (2.46) the following is taking place

$F_{2}(a, c)=f(b) \cdot F_{1}(a, c)<e(a, c) \mid a \neq b \neq c \quad$ and $\quad F_{2}(a, c)=e(a, c) \mid a=b=c$. In other words, we are getting the proof of the fact that

$F_{2}(a, c) \leq F_{1}(a, c) \Leftrightarrow e(a, b) \wedge e(b, c) \leq e(a, c) \mid \forall a, b, c \in L[0,1]$ (Q.E.D.). Note that relation $e(a, b)$ is called a fuzzy equality relation, if additionally, separability holds, i.e., $e(a, b)=1 \Leftrightarrow a=b$, then let us define a pseudo metric distance $d(a, b)$ for domain $M$, normalized to 1 , as

$$
e(a, b)=1-d(a, b)
$$

From (2.44) we are getting

$$
d(a, b)=\left\{\begin{array}{l}
(1-a) \cdot b, a>b \\
0, a=b \\
(1-b) \cdot a, b>a
\end{array}\right.
$$

\subsubsection{Approximate Fuzzy Equivalence Relations}

In [3] it was mentioned, that graduated equality of extended lines compels graduated equality of extended points. Figure 3(a) sketches a situation where two extended lines $L$ and $M$ intersect in an extended point $P$. If a third extended line $L^{\prime}$ is very similar to $L$, its intersection with $M$ yields an extended point $P$ which is very similar to $P$. It is desirable to model this fact. To do so, it is necessary 


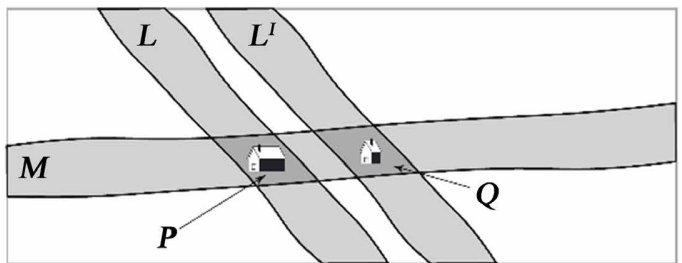

(a)

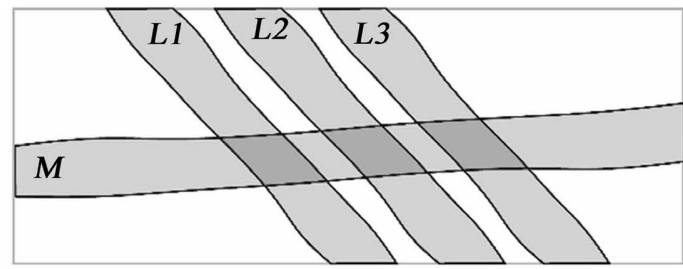

(b)

Figure 3. (a) Graduated equality of extended lines compels graduated equality of extended points. (b) Equality of extended lines is not transitive.

to allow graduated equality of extended points.

Figure 3(b) illustrates that an equality relation between extended objects need not be transitive. This phenomenon is commonly referred to as the Poincare paradox. The Poincare paradox is named after the famous French mathematician and theoretical physicist Henri Poincare, who repeatedly pointed this fact out, e.g., in [3], referring to indiscernibility in sensations and measurements. Note that this phenomenon is usually insignificant, if positional uncertainty is caused by stochastic variability. In measurements, the stochastic variability caused by measurement inaccuracy is usually much greater than the indiscernibility caused by limited resolution. For extended objects, this relation is reversed: The extension of an object can be interpreted as indiscernibility of its contributing points. In the present paper we assume that the extension of an object is being compared with the indeterminacy of its boundary. Then in [3] we also shown, that for modelling the Poincare paradox we can replace a graduated context transitivity by a weaker form:

$$
e(a, b) \wedge e(b, c) \wedge \operatorname{dis}(b) \leq e(a, c)
$$

Here dis: $M \rightarrow[0,1]$ is a lower-bound measure (discernibility measure) for the degree of transitivity that is permitted by $q$. A pair ( $e$, dis) that is reflexive, symmetric and weakly transitive (2.50) is called an approximate fuzzy $\wedge$ -equivalence relation. Let us rewrite (2.50) as follows

$$
F_{2}(a, c) \wedge \operatorname{dis}(b) \leq F_{1}(a, c)
$$

where $F_{2}(a, c), F_{1}(b, c)$ are defined in (2.46) and (2.45) correspondingly and given (2.47) we found that

$$
\operatorname{dis}(b) \equiv b \cdot(1-b)
$$

Since $\forall a \in[0,1] \Rightarrow \operatorname{dis}(a) \in[0,0.25]$, therefore (2.51) holds.

In [3] we also mentioned that an approximate fuzzy $\wedge$-equivalence relation is the upside-down version of a so-called pointless pseudo metric space $(\delta, s)$ :

$$
\begin{aligned}
& \delta(a, a)=0 \\
& \delta(a, b)=\delta(b, a) \\
& \delta(a, b) \vee \delta(b, c) \vee s(b) \geq \delta(a, c)
\end{aligned}
$$

Here, $\delta: M \rightarrow \mathfrak{R}^{+}$is a (not necessarily metric) distance between extended regions, and $s: M \rightarrow \mathfrak{R}^{+}$is a size measure and we are using an operation dis- 
junction (2.11) also shown in Table 1 . Inequality $\delta(b, c) \vee s(b) \geq \delta(a, c)$ is a weak form of the triangle inequality. It corresponds to the weak transitivity (2.50) of the approximate fuzzy $\wedge$-equivalence relation $e$. In case the size of the domain $M$ is normalized to $1, e$ and $d i s$ can be represented by [3]

$$
e(a, b)=1-\delta(a, b), \operatorname{dis}(b)=1-s(b)
$$

Note, that $\forall a \in[0,1] \Rightarrow s(a) \in[0.75,1]$

\section{PROPOSITION 10.}

If a distance between extended regions $\delta(a, b)$ from (2.53) and pseudo metric distance $d(a, b)$ for domain $M$, normalized to 1 be the same, i.e. $\delta(a, b)=d(a, b)$, then inequality $\delta(a, b) \vee \delta(b, c) \vee s(b) \geq \delta(a, c)$ holds.

Proof:

From (2.50), applying De Morgan's rule we have:

$$
\neg(\neg e(a, b) \vee \neg e(b, c) \vee \neg \operatorname{dis}(b)) \leq e(a, c)
$$

And from (2.55) we are getting

$$
\begin{gathered}
\neg(\delta(a, b) \vee \neg \delta(b, c) \vee s(b)) \leq e(a, c), \\
\text { or }(\delta(a, b) \vee \delta(b, c) \vee s(b)) \geq \neg e(a, c)
\end{gathered}
$$

Therefore, we've gotten

$$
\delta(a, b) \vee \delta(b, c) \vee s(b) \geq \delta(a, c) \text { (Q.E.D.). }
$$

But as it was mentioned in [3], given a pointless pseudo metric space $(\delta, s)$ for extended regions on a normalized domain, equations (2.57) define an approximate fuzzy $\wedge$-equivalence relation (e,dis) by simple logical negation. The so defined equivalence relation on the one hand complies with the Poincare paradox, and on the other hand retains enough information to link two extended points (or lines) via a third. For used fuzzy logic an example of a pointless pseudo metric space is the set of extended points with the following measures:

$$
\begin{gathered}
\delta(A, B):=\inf \{d(a, b) \mid a \in A, b \in B\}, \\
s(A):=\sup \{d(a, b) \mid a, b \in A\},
\end{gathered}
$$

It is easy to show that (2.58) and (2.59) are satisfied, because from (2.49) $d(a, b) \in[0,1] \mid \forall c, a, b \in[0,1]$. A pointless metric distance of extended lines can be defined in the dual space [3]:

$$
\begin{gathered}
\delta(L, M):=\inf \left\{d\left(l^{\prime}, m^{\prime}\right) \mid l \in L, m \in M\right\}, \\
s(L):=\sup \left\{d\left(l^{\prime}, m^{\prime}\right) \mid l, m \in L\right\},
\end{gathered}
$$

\subsubsection{Boundary Conditions for Granularity}

As it was mentioned in [3], in exact coordinate geometry, points and lines do not have size. Therefore, distance of points does not matter in the formulation of Euclid's first postulate. If points and lines are allowed to have extension, both, size and distance matter. Figure 4 depicts the location constraint on an extended 
line $L$ that is incident with the extended points $A$ and $B$.

The location constraint can be interpreted as tolerance in the position of $L$. In Figure 4(a) the distance of $A$ and $B$ is large with respect to the sizes of $A$ and $B$, and with respect to the width of $L$. The resulting positional tolerance for $L$ is small. In Figure 4(b), the distance of $A$ and $B$ is smaller than it is in Figure 4(a). As a consequence, the positional tolerance for $L$ becomes larger. In Figure $4(\mathrm{~b})$, $A$ and $B$ have the same distance than in Figure 4(a), but their sizes are increased. Again, positional tolerance of $L$ increases. Therefore, a formalization of Euclid's first postulate for extended primitives must take all three parameters into account: the distance of the extended points, their size, and the size of the incident line.

Figure 5 illustrates this case: Despite the fact that $A$ and $B$ are distinct extended points that are both incident with $L$, they do not specify any directional constraint for $L$. Consequently, the directional parameter of the extended lines $L$ and $L^{\prime}$ in Figure 5 may assume its maximum (at $90^{\circ}$ ). If we measure similarity (i.e., graduated equality) as inverse to distance, and if we establish a distance measure between extended lines that depends on all parameters of the line's parameter space, then $L$ and $L^{\prime}$ in Figure 5 must have maximum distance. In other words, their degree of equality is zero, even though they are distinct and incident with $A$ and $B$. The above observation can be interpreted as granularity. If we interpret the extended line $L$ in Figure 5 as a sensor, then the extended points $P$ and $Q$ are indiscernible for $L$. Note that in this context grain size is not constant but depends on the line that serves as a sensor. Based on above mentioned a granularity enters Euclid's first postulate, if points and lines have extension: If

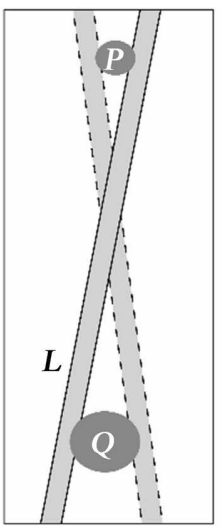

(a)

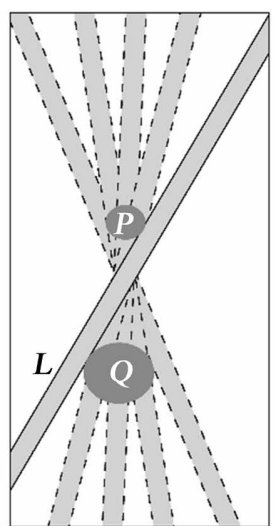

(b)

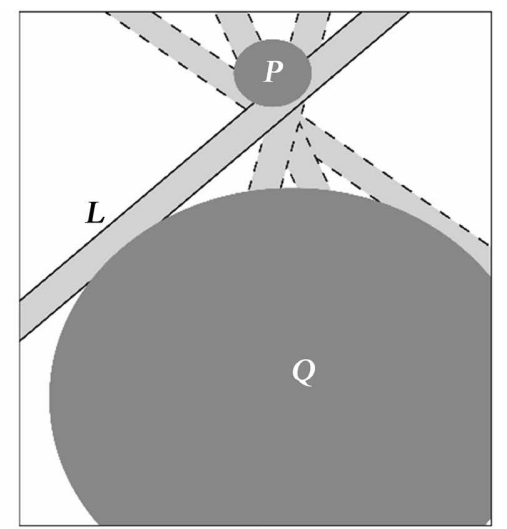

(c)

Figure 4. Size and distance matter.

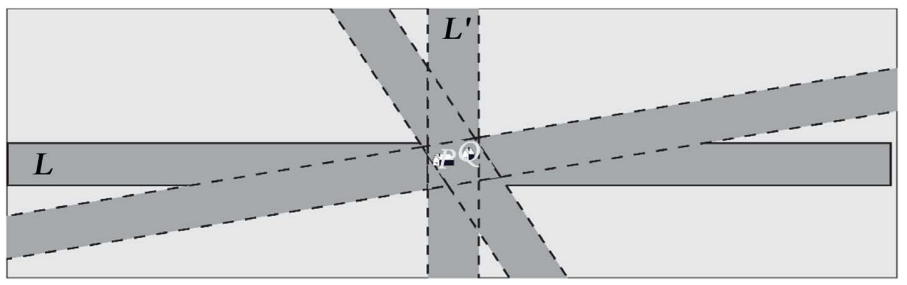

Figure 5. A and B are indiscernible for L. 
two extended points $P$ and $Q$ are too close and the extended line $L$ is too broad, then $P$ and $Q$ are indiscernible for $L$. Since this relation of indiscernibility (equality) depends not only on $P$ and $Q$, but also on the extended line $L$, which acts as a sensor, we denote it by $e(P, Q)[L]$, where $L$ serves as an additional parameter for the equality of $P$ and $Q$. In [3] the following three boundary conditions to specify a reasonable behavior of $e(P, Q)[L]$ were proposed:

1) If $s(L) \geq \delta(P, Q)+s(P)+s(Q)$, then $P$ and $Q$ impose no direction constraint on $L$ (cf. Figure 5), i.e., $P$ and $Q$ are indiscernible for $L$ to degree 1 : $e(P, Q)[L]=1$.

2) If $s(L)<\delta(P, Q)+s(P)+s(Q)$, then $P$ and $Q$ impose some direction constraint on $L$, but in general do not fix its location unambiguously. Accordingly, the degree of indiscernibility of $P$ and $Q$ lies between zero and one: $0<e(P, Q)[L]<=1$.

3) If $s(L)<\delta(P \backslash P, Q)+s(P)+s(Q)$ and $P=p, Q=q$ and $L=I$ is crisp, then $s(L)=s(P)=s(Q)=0$. Consequently, $p$ and $q$ determine the direction of $I$ unambiguously, and all positional tolerance disappears. For this case we demand $e(P, Q)[L]=0$.

In this paper we are proposing an alternative approach to one from [4] to model granulated equality.

PROPOSITION 11.

If Fuzzy Equivalence Relation $e(A, B)$ is defined in (2.52) and the width $s(L)$ of extended line $L$ is defined in (2.61), then $e(A, B)[L]$, the degree of indiscernibility of $A$ and $B$ could be calculated as follows:

$$
e(A, B)[L] \equiv e(A, B) \wedge s(L),
$$

And would satisfy a reasonable behavior, defined in $1-2$. Here $\wedge$ is conjunction operator from Table 1.

Proof:

From (2.9), (2.62) and (2.44) we have:

$$
\begin{aligned}
e(A, B)[L] & \equiv e(A, B) \wedge s(L) \\
& =\left\{\begin{array}{l}
e(A, B) \cdot s(L), e(A, B)+s(L)>1, \\
0, e(A, B)+s(L) \leq 1
\end{array}\right.
\end{aligned}
$$

but from (2.44)

$$
e(A, B)=\left\{\begin{array}{l}
(1-a) \cdot b, a<b, \\
1, a=b \\
(1-b) \cdot a, b<a,
\end{array},\right.
$$

therefore, we have the following:

1) If $A$ and $B$ impose no direction constraint on $L$ which means that $s(L)=1$ and $\delta(A, b)=0 \Rightarrow e(A, B)=1$, then $e(A, B)[L]=1$ (proof of point 1).

2) If $A$ and $B$ impose some direction constraint on $L$, but in general do not fix its location unambiguously, then from (2.62)-(2.64) we are getting 
$e(A, B)[L]=\left\{\begin{array}{l}(1-a) \cdot b, \cdot s(L),(1-a) \cdot b+s(L)>1, a<b, \\ 0,(1-a) \cdot b+s(L) \leq 1, \\ (1-b) \cdot a \cdot s(L),(1-a) \cdot b+s(L)>1, b<a,\end{array} \in(0,1) \quad\right.$ (proof of point 2).

3) If $A=a, B=b$ and $L=I$ are crisp, which means that values of $a$ and $b$ are either 0 or 1 and since $s(L)=0$, then $e(A, B)[L]=0$ (proof of point 3).

\section{Fuzzification of Euclid's First Postulate}

\subsection{A Euclid's First Postulate Formalization}

In previous chapter we identified and formalized a number of new qualities that enter into Euclid's first postulate, if extended geometric primitives are assumed. We are now in the position of formulating a fuzzified version of Euclid's first postulate. To do this, we first split the postulate

"Two distinct points determine a line uniquely."

into two sub sentences:

"Given two distinct points, there exists at least one line that passes through them."

"If more than one line passes through them, then they are equal."

These sub sentences can be formalized in Boolean predicate logic given $R_{\text {inc }}$ from (2.40) as follows

$$
\begin{gathered}
\forall a, b, \exists l,\left[R_{\text {inc }}(a, l) \wedge R_{\text {inc }}(b, l)\right] \\
\forall a, b, l, m[\neg(a=b)] \wedge\left[R_{\text {inc }}(a, l) \wedge R_{\text {inc }}(b, l)\right] \\
\wedge\left[R_{\text {inc }}(a, m) \wedge R_{\text {inc }}(b, m)\right] \rightarrow(l=m)
\end{gathered}
$$

A verbatim translation of (3.4) and (3.5) into the syntax of a fuzzy logic we use yields

$$
\begin{gathered}
\inf _{A, B} \sup _{L}\left[R_{\text {inc }}(A, L) \wedge R_{\text {inc }}(B, L)\right] \\
\inf _{A, B, L, M}\left\{[\neg e(A, B)] \wedge\left[R_{\text {inc }}(A, L) \wedge R_{\text {inc }}(B, L)\right]\right. \\
\left.\wedge\left[R_{\text {inc }}(A, M) \wedge R_{\text {inc }}(B, M)\right] \rightarrow e(L, M)\right\}
\end{gathered}
$$

where $A, B$ denote extended points, $L, M$ denote extended lines. The translated existence property (3.6) can be adopted as it is, but the translated uniqueness property (3.7) must be adapted to include granulated equality of extended points. In contrast to the Boolean case, the degree of equality of two given extended points is not constant but depends on the extended line that acts as a sensor. Consequently, the term $\neg e(A, B)$ on the left-hand side of (3.7) must be replaced by two terms, $\neg e(A, B)[L]$ and $\neg e(A, B)[M]$, one for each line, $L$ and $M$, respectively:

$$
\begin{aligned}
& \inf _{A, B, L, M}\left\{[\neg e(A, B)[L] \wedge \neg e(A, B)[M]] \wedge\left[R_{\text {inc }}(A, L) \wedge R_{\text {inc }}(B, L)\right]\right. \\
& \left.\wedge\left[R_{\text {inc }}(A, M) \wedge R_{\text {inc }}(B, M)\right] \rightarrow e(L, M)\right\}
\end{aligned}
$$


We have to use weak transitivity of graduated equality. For this reason, the discernibility measure of extended connection $\bar{A} \bar{B}$ between extended points $A$ and $B$ must be added into (3.8)

$$
\begin{aligned}
& \inf _{A, B, L, M}\left\{[\neg e(A, B)[L] \wedge \neg e(A, B)[M] \wedge \operatorname{dis}(\bar{A} \bar{B})] \wedge\left[R_{\text {inc }}(A, L) \wedge R_{\text {inc }}(B, L)\right]\right. \\
& \left.\wedge\left[R_{\text {inc }}(A, M) \wedge R_{\text {inc }}(B, M)\right] \rightarrow e(L, M)\right)
\end{aligned}
$$

But from (2.44) we get

$$
\neg e(A, B)[L]=\neg e(A, B) \wedge s(L)=\left\{\begin{array}{l}
\neg e(A, B) \cdot s(L), \neg e(A, B)+s(L)>1, \\
0, \neg e(A, B)+s(L) \leq 1
\end{array}\right.
$$

and

$\neg e(A, B)[M]=\neg e(A, B) \wedge s(M)=\left\{\begin{array}{l}\neg e(A, B) \cdot s(M), \neg e(A, B)+s(M)>1, \\ 0, \neg e(A, B)+s(M) \leq 1\end{array}\right.$

By using (3.10) and (3.11) in (3.9) we get

$$
\begin{aligned}
& \neg e(A, B)[L] \wedge \neg e(A, B)[M] \\
& =\left\{\begin{array}{l}
\neg e(A, B) \cdot s(L) \cdot \neg e(A, B) \cdot s(M), 2 \cdot \neg e(A, B)+s(L)+s(M)>1, \\
0,2 \cdot \neg e(A, B)+s(L)+s(M) \leq 1
\end{array}\right.
\end{aligned}
$$

Since from (2.40) we have

$\left[R_{\text {inc }}(A, L) \wedge R_{\text {inc }}(B, L)\right] \wedge\left[R_{\text {inc }}(A, M) \wedge R_{\text {inc }}(B, M)\right] \equiv 1$, then (3.9) could be rewritten as follows

$$
\inf _{A, B, L, M}\{[\neg e(A, B)[L] \wedge \neg e(A, B)[M] \wedge \operatorname{dis}(\bar{A} \bar{B})] \wedge 1 \rightarrow e(L, M)\}
$$

It means that the "sameness" of extended lines $e(L, M)$ depends on $[\neg e(A, B)[L] \wedge \neg e(A, B)[M] \wedge \operatorname{dis}(\bar{A} \bar{B})]$ only and could be calculated by (3.12) and (2.52) respectively.

\subsection{Fuzzy Logical Inference for Euclid's First Postulate}

Similarly, to an approach from [3], we suggest to use the same fuzzy logic (Table 1) and correspondent logical inference [5] [6] [7] and [8] to determine the value of $e(L, M)$. For this purpose, let us represent a value of following $E(a, b, l, m)=\neg e(A, B)[L] \wedge \neg e(A, B)[M]$ from (3.12) and $D(a, b)=\operatorname{dis}(\bar{A}, \bar{B})$ from (2.52) functions. Note, that values from both $E(a, b, l, m) \in\left[E_{\min }, E_{\max }\right]$ and $D(a, b) \in\left[D_{\min }, D_{\max }\right]$. In our case $E(a, b, l, m) \in[0,1] \quad D(a, b) \in[0,0.25]$. We represent $E$ as a fuzzy set forming linguistic variable, described by a triplet of the form $E=\left\{\left\langle E_{i}, X, \tilde{E}\right\rangle\right\}, E_{i} \in T(x)$, $\forall i \in[0, \operatorname{Card} X]$, where $T_{i}(x)$ is extended term set of the linguistic variable "degree of indiscernibility" from Table 3, $\tilde{E}$ is normal fuzzy set represented by membership function $\mu_{E}: X \rightarrow[0,1]$, where $X=\{0,1,2, \cdots, 10\}$ universe set and $\operatorname{CardX}$ is power set of the set $U$. We will use the following mapping $\alpha: \tilde{E} \rightarrow X\left|x_{i}=\operatorname{Ent}\left[(\operatorname{Card} X-1) \times E_{i}\right]\right| \forall i \in[0, \operatorname{CardX}]$, where 


$$
\tilde{E}=\int_{X} \mu_{E}(x) / x
$$

To determine the estimates of the membership function in terms of singletons from (3.14) in the form $\mu_{E}\left(x_{i}\right) / x_{i} \mid \forall i \in[0, \operatorname{Card} X]$ we propose the following procedure.

$$
\begin{aligned}
& \forall i \in[0, \operatorname{Card} X], \forall E_{i} \in[0,1], \\
& \mu\left(x_{i}\right)=1-\frac{1}{\operatorname{Card} X-1} \times \mid x_{i}-\operatorname{Ent}\left[(\operatorname{Card} X-1) \times E_{i}\right],
\end{aligned}
$$

We also represent $D$ as a fuzzy set forming linguistic variable, described by a triplet of the form $D=\left\{\left\langle D_{j}, Y, \tilde{D}\right\rangle\right\}, D_{j} \in T(y), \forall j \in[0, \operatorname{Card} Y]$, where $T_{j}(y)$ is extended term set of the linguistic variable "discernibility measure "from Table 3, $\tilde{D}$ is normal fuzzy set represented by membership function $\mu_{D}: Y \rightarrow[0,1]$

We will use the following mapping

$$
\begin{gathered}
\beta: \tilde{D} \rightarrow Y\left|y_{j}=\operatorname{Ent}\left[(\operatorname{Card} Y-1) \times D_{j}\right]\right| \forall j \in[0, \operatorname{Card} Y], \text { where } \\
\tilde{D}=\int_{Y} \mu_{D}(y) / y
\end{gathered}
$$

On the other hand, to determine the estimates of the membership function in terms of singletons from (3.16) in the form $\mu_{D}\left(y_{j}\right) / y_{j} \mid \forall j \in[0, \operatorname{CardY}]$ we propose the following procedure.

$$
\begin{aligned}
& \forall j \in[0, \operatorname{Card} Y], \forall D_{j} \in[0,0.25], \\
& \mu\left(y_{j}\right)=1-\frac{1}{\operatorname{Card} Y-1} \times\left|y_{j}-\operatorname{Ent}\left[(\operatorname{Card} Y-1) \times D_{j} / 0.25\right]\right|,
\end{aligned}
$$

Let us represent $e(L, M)$ as a fuzzy set $\tilde{S}$, forming linguistic variable, described by a triplet of the form $S=\left\{\left\langle S_{k}, Z, \tilde{S}\right\rangle\right\}, S_{k} \in T(z), \forall k \in[0$, CardZ $]$, where $T_{k}(z)$ is extended set term set of the linguistic variable "extended lines sameness" from Table 3. $\tilde{S}$ is normal fuzzy set represented by membership function $\mu_{s}: Z \rightarrow[0,1]$, where $Z=\{0,1,2, \cdots, 10\}$ universe set and CardZ is power set of the set $Z$. We will use the following mapping

$$
\begin{gathered}
\gamma: \tilde{S} \rightarrow Z\left|z_{k}=\operatorname{Ent}\left[(\operatorname{CardZ}-1) \times S_{k}\right]\right| \forall k \in[0, \text { CardZ }], \text { were } \\
\tilde{S}=\int_{Z} \mu_{s}(z) / z
\end{gathered}
$$

Again, to determine the estimates of the membership function in terms of singletons from (3.18) in the form $\mu_{S}\left(z_{k}\right) / z_{k} \mid \forall k \in[0, \operatorname{CardZ}]$ we propose the following procedure.

$$
\begin{aligned}
& \forall k \in[0, \operatorname{CardZ}], \forall S_{k} \in[0,1], \\
& \mu\left(z_{k}\right)=1-\frac{1}{\operatorname{CardZ}-1} \times \mid z_{k}-\operatorname{Ent}\left[(\operatorname{CardZ}-1) \times S_{k}\right],
\end{aligned}
$$

To get an estimates of values of $e(L, M)$ or "extended lines sameness", represented by fuzzy set $\tilde{S}$ from (3.18) given the values of $E(a, b, l, m)$ or "degree of indiscernibility" and $D(a, b)$ "discernibility measure", represented by fuzzy sets $\tilde{E}$ from (3.14) and $\tilde{D}$ from (3.16) respectively, we will use a Fuzzy Conditional 
Table 3. Linguistic variables in fuzzy logical inference for Euclid's first postulate.

\begin{tabular}{|c|c|c|c|}
\hline \multicolumn{3}{|c|}{ Value of variable } & \multirow{2}{*}{$\begin{array}{c}x_{i} \in X, y_{j} \in Y, z_{k} \in Z \\
\forall i, j, k \in[0,10]\end{array}$} \\
\hline "Degree of indiscernibility" & "Discernibility measure" & "Extended lines sameness" & \\
\hline Lowest & highest & nothing in common & 0 \\
\hline very low & almost highest & very far & 1 \\
\hline Low & high & far & 2 \\
\hline bit higher than low & pretty high & bit closer than far & 3 \\
\hline almost average & bit higher than average & almost average distance & 4 \\
\hline Average & average & average & 5 \\
\hline bit higher than average & almost average & bit closer than average & 6 \\
\hline pretty high & bit higher than low & pretty close & 7 \\
\hline High & low & close & 8 \\
\hline almost highest & very low & almost the same & 9 \\
\hline Highest & lowest & the same & 10 \\
\hline
\end{tabular}

Inference Rule, formulated by means of "common sense" as a following conditional clause:

$$
P=\text { "IF ( } \tilde{E} \text { is } X) \operatorname{AND}(\tilde{D} \text { is } Y) \text {, THEN ( } \tilde{S} \text { is } Z) \text { " }
$$

In other words, we use fuzzy conditional inference of the following type [7]:

Ant 1: If $e$ is $E$ and $d$ is $D$ then $s$ is $S$

Ant2: $e$ is $E^{\prime}$ and $d$ is $D^{\prime}$

Cons: $s$ is $S^{\prime}$

where $E, E^{\prime} \subseteq X, D, D^{\prime} \subseteq Y$ and $S, S^{\prime} \subseteq Z$.

Now for fuzzy sets (3.14), (3.16) and (3.18) a binary relationship for the fuzzy conditional proposition of the type (3.20) and (3.21) for fuzzy logic we use so far is defined as

$$
\begin{aligned}
& R\left(A_{1}(e, d), A_{2}(s)\right)=[E \times X \cap D \times Y] \\
& \rightarrow Z \times S=\int_{X \times Y \times Z}\left(\left[\mu_{E}(x) / x \wedge \mu_{D}(y) / y\right] \rightarrow \mu_{S}(z)\right) /(x, y, z)
\end{aligned}
$$

Given (2.10) and since we consider that $\operatorname{CardX}=\operatorname{Card} Y=\operatorname{CardZ}$, then expression (3.22) looks like

$$
\begin{aligned}
& {\left[\mu_{E}(x) \wedge \mu_{D}(y)\right]} \\
& \rightarrow \mu_{S}(z)=\left\{\begin{array}{l}
\left(1-\left[\mu_{E}(x) \wedge \mu_{D}(y)\right]\right) \cdot \mu_{S}(z),\left[\mu_{E}(x) \wedge \mu_{D}(y)\right]>\mu_{S}(z), \\
1,\left[\mu_{E}(x) \wedge \mu_{D}(y)\right] \leq \mu_{S}(z) .
\end{array}\right.
\end{aligned}
$$

where $\left[\mu_{E}(x) \wedge \mu_{D}(y)\right]$ is $\min \left[\mu_{E}(x), \mu_{D}(y)\right]$. It is well known that given a unary relationship $R\left(A_{1}(e, d)\right)=E^{\prime} \cap D^{\prime}$ one can obtain the consequence $R\left(A_{2}(e)\right)$ by applying compositional rule of inference (CRI) to $R\left(A_{1}(e, d)\right)$ 
and $R\left(A_{1}(e, d), A_{2}(e)\right)$ of type (3.22):

$$
\begin{aligned}
& R\left(A_{2}(s)\right)=E^{\prime} \cap D^{\prime} \circ R\left(A_{1}(e, d), A_{2}(s)\right) \\
& =\int_{X \times Y}\left[\mu_{E^{\prime}}(x) \wedge \mu_{D^{\prime}}(y)\right] /(x, y) \circ \int_{X \times Y \times Z}\left[\mu_{E}(x) \wedge \mu_{D}(y)\right] \rightarrow \mu_{S}(z) /(x, y, z)(3.24) \\
& =\int_{Z} \bigcup_{x \in X, y \in Y}\left\{\left[\mu_{E^{\prime}}(x) \wedge \mu_{D^{\prime}}(y)\right] \wedge\left(\left[\mu_{E}(x) \wedge \mu_{D}(y)\right] \rightarrow \mu_{S}(z)\right)\right\} / z
\end{aligned}
$$

But for practical purposes we will use another Fuzzy Conditional Rule (FCR)

$$
\begin{aligned}
& R\left(A_{1}(e, d), A_{2}(s)\right)=(P \times U \rightarrow V \times S) \cap(\neg P \times U \rightarrow V \times \neg S) \\
& =\int_{U \times V}\left(\mu_{P}(u) \rightarrow \mu_{S}(v)\right) \wedge\left(\left(1-\mu_{P}(u)\right) \rightarrow\left(1-\mu_{S}(v)\right)\right) /(u, v)
\end{aligned}
$$

where $P=E \cap D$ and $U=X=Y$, therefore from (3.25) we are getting

$$
\begin{aligned}
R\left(A_{1}(e, d), A_{2}(s)\right)= & \left(\mu_{P}(u) \rightarrow \mu_{S}(v)\right) \wedge\left(\left(1-\mu_{P}(u)\right) \rightarrow\left(1-\mu_{S}(v)\right)\right) \\
= & \left\{\begin{array}{l}
\left(1-\mu_{P}(u)\right) \cdot \mu_{S}(v), \mu_{P}(u)>\mu_{S}(v), \\
1, \mu_{P}(u)=\mu_{S}(v), \\
\left(1-\mu_{S}(v)\right) \cdot \mu_{P}(u), \mu_{P}(u)<\mu_{S}(v) .
\end{array}\right.
\end{aligned}
$$

The FCR from (3.26) gives more reliable results.

\subsection{Example}

To build a binary relationship matrix of type (3.25) we us use a conditional clause of type (3.20):

$P=$ "IF ( $S$ is lowest") AND ( $D$ is "highest '), THEN ( $E$ is "nothing in common") (3.27)

To build membership functions for fuzzy sets $S, D$ and $E$ we use (3.15), (3.17) and (3.19) respectively.

In (3.27) the membership functions for fuzzy set $S$ (for instance) would look like:

$$
\begin{aligned}
\mu_{s}(\text { "lowest" })= & 1 / 0+0.9 / 1+0.8 / 2+0.7 / 3+0.6 / 4+0.5 / 5 \\
& +0.4 / 6+0.3 / 7+0.2 / 8+0.1 / 9+0 / 10
\end{aligned}
$$

Same membership functions we use for fuzzy sets $D$ and $E$.

From (3.26) we have $R\left(A_{1}(s, d), A_{2}(e)\right)$ from Table 4.

Suppose from (3.12) a current estimate of $E(a, b, l, m)=0.6$ and from (2.62) $D(a, b)=0.25$. By using (3.15) and (3.17) respectively we got (see Table 2 )

$$
\begin{gathered}
\mu_{E} \text { ("bit higher than average") } \\
=0.4 / 0+0.5 / 1+0.6 / 2+0.7 / 3+0.8 / 4+0.9 / 5 \\
+1 / 6+0.9 / 7+0.8 / 8+0.7 / 9+0.6 / 10 \\
\mu_{D} \text { ("pretty high") }=0.7 / 0+0.8 / 1+0.9 / 2+1 / 3+0.9 / 4+0.8 / 5 \\
+0.7 / 6+0.6 / 7+0.5 / 8+0.4 / 9+0.3 / 10
\end{gathered}
$$

It is apparent that:

$$
\begin{aligned}
R\left(A_{1}\left(s^{\prime}, d^{\prime}\right)\right)= & \mu_{E}(u) \wedge \mu_{D}(u) \\
= & 0.4 / 0+0.5 / 1+0.6 / 2+0.7 / 3+0.8 / 4+0.8 / 5 \\
& +0.7 / 6+0.6 / 7+0.5 / 8+0.4 / 9+0.3 / 10
\end{aligned}
$$


Table 4. Binary relationship matrix of a current example.

\begin{tabular}{cccccccccccc}
\hline$p \rightarrow s$ & 1 & 0.9 & 0.8 & 0.7 & 0.6 & 0.5 & 0.4 & 0.3 & 0.2 & 0.1 & 0 \\
\hline 1 & 1 & 0 & 0 & 0 & 0 & 0 & 0 & 0 & 0 & 0 & 0 \\
0.9 & 0 & 1 & 0.08 & 0.07 & 0.06 & 0.05 & 0.04 & 0.03 & 0.02 & 0.01 & 0 \\
0.8 & 0 & 0.08 & 1 & 0.14 & 0.12 & 0.1 & 0.08 & 0.06 & 0.04 & 0.02 & 0 \\
0.7 & 0 & 0.07 & 0.14 & 1 & 0.18 & 0.15 & 0.12 & 0.09 & 0.06 & 0.03 & 0 \\
0.6 & 0 & 0.06 & 0.12 & 0.18 & 1 & 0.2 & 0.16 & 0.12 & 0.08 & 0.04 & 0 \\
0.5 & 0 & 0.05 & 0.1 & 0.15 & 0.2 & 1 & 0.2 & 0.15 & 0.1 & 0.05 & 0 \\
0.4 & 0 & 0.04 & 0.08 & 0.12 & 0.16 & 0.2 & 1 & 0.18 & 0.12 & 0.06 & 0 \\
0.3 & 0 & 0.03 & 0.06 & 0.09 & 0.12 & 0.15 & 0.18 & 1 & 0.14 & 0.07 & 0 \\
0.2 & 0 & 0.02 & 0.04 & 0.06 & 0.08 & 0.1 & 0.12 & 0.14 & 1 & 0.08 & 0 \\
0.1 & 0 & 0.01 & 0.02 & 0.03 & 0.04 & 0.05 & 0.06 & 0.07 & 0.08 & 1 & 0 \\
0 & 0 & 0 & 0 & 0 & 0 & 0 & 0 & 0 & 0 & 0 & 1 \\
\hline
\end{tabular}

By applying compositional rule of inference (CRI) to $R\left(A_{1}\left(s^{\prime}, d^{\prime}\right)\right)$ and $R\left(A_{1}(s, d), A_{2}(e)\right)$ from Table 4.

$$
\begin{aligned}
& R\left(A_{2}\left(e^{\prime}\right)\right)=R\left(A_{1}\left(s^{\prime}, d^{\prime}\right)\right) \circ R\left(A_{1}(s, d), A_{2}(e)\right) \text { we got the following: } \\
& R\left(A_{2}\left(e^{\prime}\right)\right)= \mu_{E}(u) \wedge \mu_{D}(u) \\
&= 0.4 / 0+0.5 / 1+0.6 / 2+0.7 / 3+0.8 / 4+0.8 / 5 \\
&+0.7 / 6+0.6 / 7+0.5 / 8+0.4 / 9+0.3 / 10
\end{aligned}
$$

It is obvious that the value of fuzzy set $S$ is laying between terms "almost average distance" and "average distance" (see Table 2), which means that approximate values for $e(L . M)$ are $e(L, M) \in[0.5,0.6]$.

\section{Logical Principles of AIA Orientation}

\subsection{Preliminary Considerations}

Let consider that both Target and Object, a subject of mutual navigation, to be presented as octagons, depicted on Figure 6. We use octagons for simplification's sake only. Given the fact that we are studying a projection-based model, both targets and objects could be presented as follows:

$T=\left\{t_{j}\right\} ; j=\overline{1, n}$. Where $j$ is number of heights of a Target, whereas $O=\left\{o_{i}\right\} ; i=\overline{1, m}$ and $i$ is number of heights of an Object. Both a target and an object could be presented in three-dimensional space as follows:

$$
t_{j} \in T=\left\{x_{j}^{t}, y_{j}^{t}, z_{j}^{t}\right\} ; j=\overline{1, n}, o_{j} \in O=\left\{x_{i}^{o}, y_{i}^{o}, z_{i}^{o}\right\} ; i=\overline{1, m} .
$$

On the other hand, from Figure 6 each value of both a Target and an Object coordinate could be presented as a pair of minimal and maximal (per 3D coordinate) values of them. For targets, in particular

$$
\begin{aligned}
& \forall j=\overline{1, n} \mid x_{\min }^{T}=\min _{j}\left\{x_{j}^{t}\right\}, x_{\max }^{T}=\max _{j}\left\{x_{j}^{t}\right\}, y_{\min }^{T}=\min _{j}\left\{y_{j}^{t}\right\}, y_{\max }^{T}=\max _{j}\left\{y_{j}^{t}\right\}, \\
& z_{\min }^{T}=\min _{j}\left\{z_{j}^{t}\right\}, z_{\max }^{T}=\max _{j}\left\{z_{j}^{t}\right\} .
\end{aligned}
$$




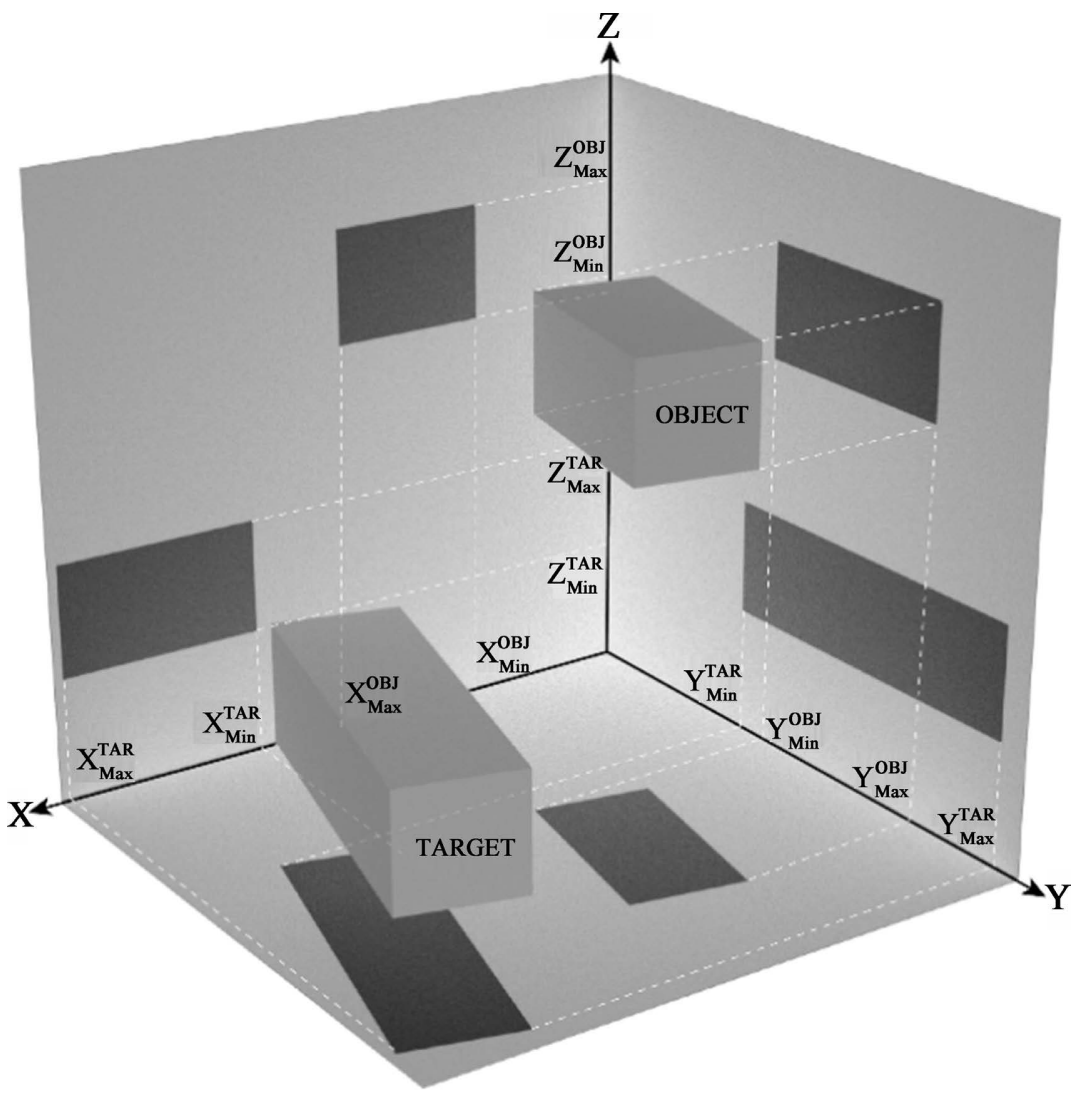

Figure 6. Object and target space representation.

By analogy, for objects we are getting:

$$
\begin{aligned}
& \forall i=\overline{1, m} \mid x_{\min }^{O}=\min _{i}\left\{x_{i}^{o}\right\}, x_{\max }^{O}=\max _{i}\left\{x_{i}^{o}\right\}, y_{\min }^{O}=\min _{i}\left\{y_{i}^{o}\right\}, y_{\max }^{O}=\max _{i}\left\{y_{i}^{o}\right\}, \\
& z_{\min }^{O}=\min _{i}\left\{z_{i}^{o}\right\}, z_{\max }^{O}=\max _{i}\left\{z_{i}^{o}\right\} .
\end{aligned}
$$

It is import to consider the following features of both target and object from Figure 6, i.e., width $(w)$, depth $(d)$ and height $(h)$ of them:

$$
\begin{aligned}
& w^{T}=\left|x_{\max }^{T}-x_{\min }^{T}\right| \cdot \tan (\theta(x, y)) ; \\
& d^{T}=\left|y_{\max }^{T}-y_{\min }^{T}\right| \cdot \tan (\theta(x, z)) ; \\
& h^{T}=\left|z_{\max }^{T}-z_{\min }^{T}\right| \cdot \tan (\theta(z, y)), \\
& w^{O}=\left|x_{\max }^{O}-x_{\min }^{O}\right| \cdot \tan (\theta(x, y)) ; \\
& d^{O}=\left|y_{\max }^{O}-y_{\min }^{O}\right| \cdot \tan (\theta(x, z)) ; \\
& h^{O}=\left|z_{\max }^{O}-z_{\min }^{O}\right| \cdot \tan (\theta(z, y)) .
\end{aligned}
$$

where $\theta(x, y), \theta(x, z)$ and $\theta(z, y)$ will be defined in (4.19).

\subsection{Predicates of Two Entities Mutual Relations}

Considering (4.2)-(4.5) we can formulate some logical predicates, which would describe mutual positioning of two players in the paradigm of a projectionbased model. Let us define predicates as relation symbols, describing a variety of 
positions of two entities in a space in a connection to each other.

\subsubsection{Size Comparison Predicates}

We define size comparison of Target and Object as the following relation symbols, given (4.4) and (4.5)

$$
\begin{aligned}
& \operatorname{LARGER}(T, O) \Rightarrow w^{T}>w^{O} \& \mid d^{T}>d^{O} \& h^{T}>h^{O}, \\
& \operatorname{SMALLER}(T, O) \Rightarrow w^{T}<w^{O} \& \mid d^{T}<d^{O} \& h^{T}<h^{O} \\
& \operatorname{LARGER}(O, T) \Rightarrow w^{O}>w^{T} \& \mid d^{O}>d^{T} \& h^{O}>h^{T}, \\
& \operatorname{SMALLER}(O, T) \Rightarrow w^{O}<w^{T} \& \mid d^{O}<d^{T} \& h^{O}<h^{T}
\end{aligned}
$$

\subsubsection{Mutual Positioning Predicates}

We also define mutual positioning of a Target and an Object from Figure 6 as the following relation symbols, given (4.2) and (4.3)

$$
\begin{aligned}
& \operatorname{HIGHER}(T, O) \Rightarrow z_{\min }^{T} \geq z_{\text {max }}^{O}, \operatorname{LOWER}(T, O) \Rightarrow z_{\min }^{T} \leq z_{\text {max }}^{O} \\
& \operatorname{HIGHER}(O, T) \Rightarrow z_{\min }^{O} \geq z_{\max }^{T}, \operatorname{LOWER}(O, T) \Rightarrow z_{\min }^{O} \leq z_{\max }^{T} \\
& \operatorname{ATLEFT}(T, O) \Rightarrow x_{\max }^{T} \leq x_{\min }^{O}, \operatorname{ATRIGHT}(T, O) \Rightarrow x_{\max }^{T} \geq x_{\min }^{O} \\
& \operatorname{ATLEFT}(O, T) \Rightarrow x_{\max }^{O} \leq x_{\min }^{T}, \operatorname{ATRIGHT}(O, T) \Rightarrow x_{\max }^{O} \geq x_{\min }^{T} \\
& \operatorname{BEHIND}(T, O) \Rightarrow y_{\max }^{T} \leq y_{\min }^{O}, \operatorname{INFRONT}(T, O) \Rightarrow y_{\max }^{T} \geq y_{\min }^{O} \\
& \operatorname{BEHIND}(O, T) \Rightarrow y_{\max }^{O} \leq y_{\min }^{T}, \operatorname{INFRONT}(O, T) \Rightarrow y_{\max }^{O} \geq y_{\min }^{T} \\
& \operatorname{ONTOP}(T, O) \Rightarrow z_{\min }^{T}=z_{\max }^{O}, \operatorname{ONBOTTOM}(T, O) \Rightarrow z_{\max }^{T}=z_{\min }^{O} \\
& \operatorname{ONTOP}(O, T) \Rightarrow z_{\min }^{O}=z_{\max }^{T}, \operatorname{ONBOTTOM}(O, T) \Rightarrow z_{\max }^{O}=z_{\min }^{T}
\end{aligned}
$$

\subsubsection{Preconditions for Actions and Entity Shape Estimation}

Before formulation of a possible actions, which could be performed by certain entities, and given (4.2) and (4.3) we have to consider for each entity the following points in 3-dimentional space $T_{\text {center }}=\left\{x_{\text {center }}^{T}, y_{\text {center }}^{T}, z_{\text {center }}^{T}\right\}$ for a Target and $O_{\text {center }}=\left\{x_{\text {center }}^{O}, y_{\text {center }}^{O}, z_{\text {center }}^{O}\right\}$ for an Object correspondingly, These points could define some conditional center of a gravity for each of them (median points in space)

$$
\begin{gathered}
x_{\text {center }}^{T}=\frac{x_{\max }^{T}+x_{\min }^{T}}{2}, x_{\text {center }}^{O}=\frac{x_{\max }^{O}+x_{\min }^{O}}{2} \\
y_{\text {center }}^{T}=\frac{y_{\max }^{T}+y_{\min }^{T}}{2}, y_{\text {center }}^{O}=\frac{y_{\max }^{O}+y_{\min }^{O}}{2} \\
z_{\text {center }}^{T}=\frac{z_{\max }^{T}+z_{\min }^{T}}{2}, z_{\text {center }}^{O}=\frac{z_{\max }^{O}+z_{\min }^{O}}{2}
\end{gathered}
$$

We use the following 3-coordinate mapping, depicted in Figure 7 to define an angel in 3D space between a Target and an Object, i.e., between points $T_{\text {center }}$ and $O_{\text {center }}$.

Where each dimensional angel (mapping) $\theta(x, y), \theta(x, z)$ and $\theta(z, y)$ is defined as follows 


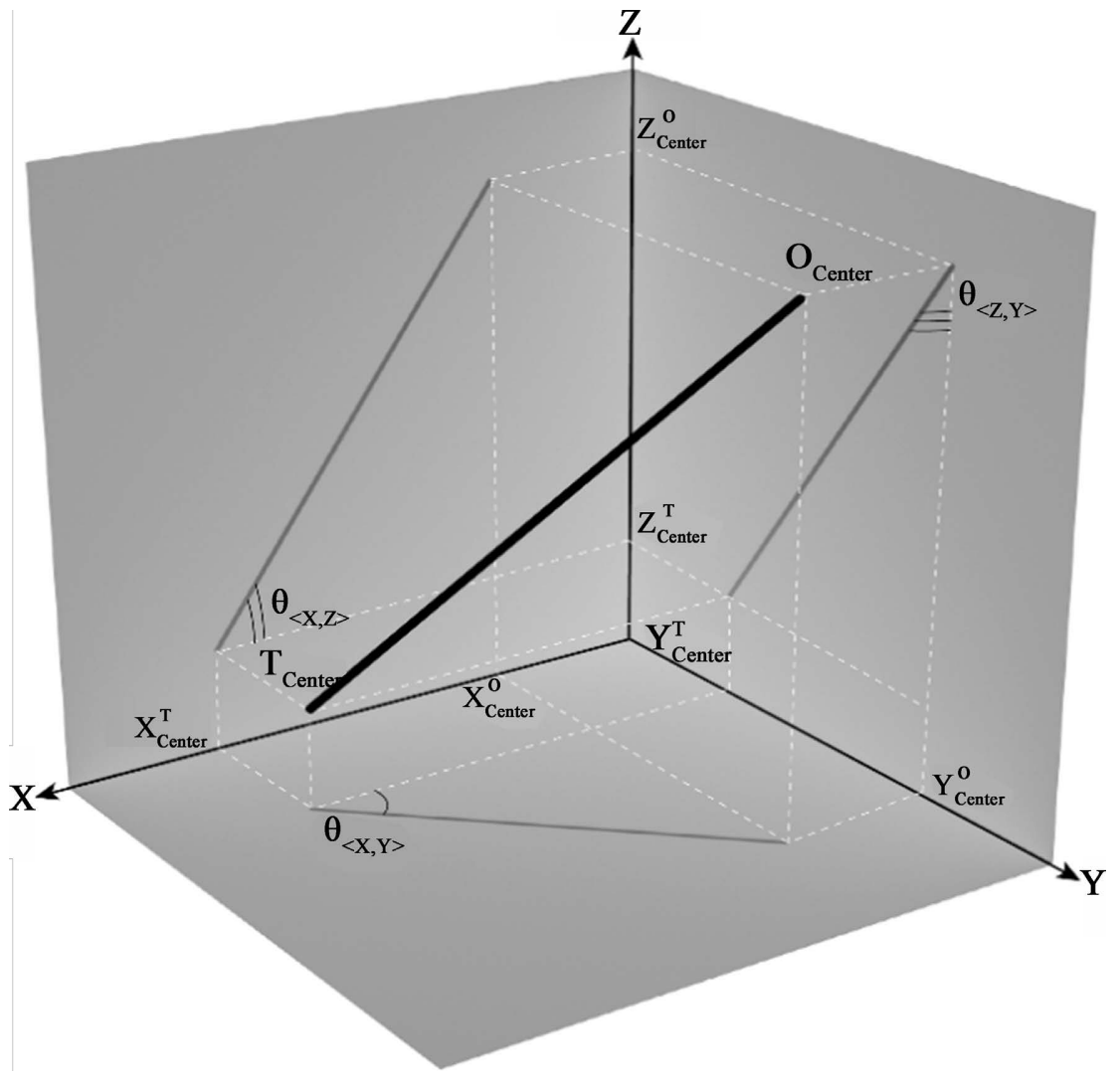

Figure 7. Angels mapping for object and target.

$$
\begin{aligned}
& \theta(x, y)=\left\{\begin{array}{l}
\frac{\pi}{2}-\arctan \left(\frac{y_{\text {center }}^{T}-y_{\text {center }}^{O}}{x_{\text {center }}^{T}-x_{\text {center }}^{O}}\right), x_{\text {center }}^{T}>x_{\text {center }}^{O}, \\
\frac{3 \pi}{2}-\arctan \left(\frac{y_{\text {center }}^{T}-y_{\text {center }}^{O}}{x_{\text {center }}^{T}-x_{\text {center }}^{O}}\right), x_{\text {center }}^{T}<x_{\text {center }}^{O} ; \\
0, x_{\text {center }}^{T}=x_{\text {center }}^{O}, y_{\text {center }}^{T}>y_{\text {center }}^{O}, \\
1, x_{\text {center }}^{T}=x_{\text {center }}^{O}, y_{\text {center }}^{T}<y_{\text {center }}^{O},
\end{array}\right. \\
& \theta(x, z)=\left\{\begin{array}{l}
\frac{\pi}{2}-\arctan \left(\frac{z_{c e n t e r}^{T}-z_{\text {center }}^{O}}{x_{\text {center }}^{T}-x_{\text {center }}^{O}}\right), x_{\text {center }}^{T}>x_{\text {center }}^{O}, \\
\frac{3 \pi}{2}-\arctan \left(\frac{z_{\text {center }}^{T}-z_{\text {center }}^{O}}{x_{\text {center }}^{T}-x_{\text {center }}^{O}}\right), x_{\text {center }}^{T}<x_{\text {center }}^{O} ; \\
0, x_{\text {center }}^{T}=x_{\text {center }}^{O}, z_{\text {center }}^{T}>y_{\text {center }}^{O}, \\
1, x_{\text {center }}^{T}=x_{\text {center }}^{O}, z_{\text {center }}^{T}<z_{\text {center }}^{O},
\end{array}\right. \\
& \theta(z, y)=\left\{\begin{array}{l}
\frac{\pi}{2}-\arctan \left(\frac{y_{\text {center }}^{T}-y_{\text {center }}^{O}}{z_{\text {center }}^{T}-z_{\text {center }}^{O}}\right), z_{\text {center }}^{T}>z_{\text {center }}^{O}, \\
\frac{3 \pi}{2}-\arctan \left(\frac{y_{\text {center }}^{T}-y_{\text {center }}^{O}}{z_{\text {center }}^{T}-z_{\text {center }}^{O}}\right), z_{\text {center }}^{T}<z_{\text {center }}^{O}, \\
0, z_{\text {center }}^{T}=z_{\text {center }}^{O}, y_{\text {center }}^{T}>y_{\text {center }}^{O}, \\
1, z_{\text {center }}^{T}=z_{\text {center }}^{O}, y_{\text {center }}^{T}<y_{\text {center }}^{O},
\end{array}\right.
\end{aligned}
$$


Let us also introduce the following values, which define entities coordinate derivation in space.

1) Vertical (Z-dimension) derivation would be presented as

$\forall x, y \mid x \in\left[x_{\text {min }}, x_{\text {max }}\right], y \in\left[y_{\text {min }}, y_{\text {max }}\right] \Rightarrow \Delta z(x, y)=z^{\max }(x, y)-z^{\min }(x, y)$

2) Horizontal (X-dimension) derivation would be presented as

$\forall z, y \mid z \in\left[z_{\min }, z_{\max }\right], y \in\left[y_{\min }, y_{\max }\right] \Rightarrow \Delta x(z, y)=x^{\max }(z, y)-x^{\min }(z, y)$

3) Y-dimension derivation would be presented as

$$
\forall x, z \mid x \in\left[x_{\min }, x_{\max }\right], z \in\left[z_{\min }, z_{\max }\right] \Rightarrow \Delta y(x, z)=y^{\max }(x, z)-y^{\min }(x, z)
$$

\subsubsection{Entity Shape Estimation Predicates}

We define the following predicates by using (4.20)-(4.22)

1) Entity has right geometric form (RGF)

$$
\begin{aligned}
& R G F(E) \Rightarrow \Delta z^{E}\left(x^{E}, y^{E}\right) \equiv \text { const } \& \Delta x^{E}\left(z^{E}, y^{E}\right) \equiv \text { const } \\
& \& \Delta y^{E}\left(x^{E}, y^{E}\right) \equiv \text { const }
\end{aligned}
$$

2) Entity has flat left and right surfaces (FLRS)

$$
\operatorname{FLRS}(E) \Rightarrow \Delta x^{E}\left(z^{E}, y^{E}\right) \equiv \text { const, } \forall z^{E}, y^{E} \mid z^{E} \in\left[z_{\text {min }}^{E}, z_{\max }^{E}\right], y^{E} \in\left[y_{\min }^{E}, y_{\max }^{E}\right]
$$

3) Entity has flat top and bottom (FTB)

$$
\operatorname{FTB}(E) \Rightarrow \Delta z^{E}\left(x^{E}, y^{E}\right) \equiv \text { const, } \forall x^{E}, y^{E} \mid x^{E} \in\left[x_{\min }^{E}, x_{\max }^{E}\right], y^{E} \in\left[y_{\min }^{E}, y_{\max }^{E}\right]
$$

4) Entity has flat front and back surfaces (FFBS)

$$
\operatorname{FFBS}(E) \Rightarrow \Delta y^{E}\left(x^{E}, z^{E}\right) \equiv \text { const }, \forall x^{E}, z^{E} \mid x^{E} \in\left[x_{\min }^{E}, x_{\max }^{E}\right], z^{E} \in\left[z_{\min }^{E}, z_{\max }^{E}\right]
$$

\subsubsection{Docking Positioning Predicates}

We define the following predicates by using (4.16)-(4.18)

1) Object docks in front of a Target (DIF)

$$
\operatorname{DIF}(O, T) \Rightarrow x_{\text {center }}^{T}=x_{\text {center }}^{O} \& z_{\text {center }}^{T}=z_{\text {center }}^{O} \& y_{\max }^{T}=y_{\text {min }}^{O}
$$

2) Object docks at back of a Target (DAB)

$$
D A B(O, T) \Rightarrow x_{\text {center }}^{T}=x_{\text {center }}^{O} \& z_{\text {center }}^{T}=z_{\text {center }}^{O} \& y_{\min }^{T}=y_{\max }^{O}
$$

3) Object docks at left of a Target (DAL)

$$
D A L(O, T) \Rightarrow y_{\text {center }}^{T}=y_{\text {center }}^{O} \& z_{\text {center }}^{T}=z_{\text {center }}^{O} \& x_{\min }^{T}=x_{\max }^{O}
$$

4) Object docks at right of a Target (DAR)

$$
\operatorname{DAR}(O, T) \Rightarrow y_{\text {center }}^{T}=y_{\text {center }}^{O} \& z_{\text {center }}^{T}=z_{\text {center }}^{O} \& x_{\text {max }}^{T}=x_{\text {min }}^{O}
$$

5) Object docks on top of a Target (DOT)

$$
D O T(O, T) \Rightarrow x_{\text {center }}^{T}=x_{\text {center }}^{O} \& y_{\text {center }}^{T}=y_{\text {center }}^{O} \& z_{\text {max }}^{T}=z_{\text {min }}^{O}
$$

6) Object docks under (at bottom) of a Target (DUN)

$$
D U N(O, T) \Rightarrow x_{\text {center }}^{T}=x_{\text {center }}^{O} \& y_{\text {center }}^{T}=y_{\text {center }}^{O} \& z_{\min }^{T}=z_{\max }^{O}
$$


It is well known fact, that distance $\boldsymbol{d}$ between two points $a, b \in \mathfrak{R}^{3}$ represents the Euclidean distance in $\mathfrak{R}^{3}$. Let us define Euclidean distance for a Target and an Object (between points $T_{\text {center }}$ and $O_{\text {center }}$ ) from Figure 7 the following way

$$
d(T, O)=\sqrt{\left(x_{\text {center }}^{T}-x_{\text {center }}^{O}\right)^{2}+\left(y_{\text {center }}^{T}-y_{\text {center }}^{O}\right)^{2}+\left(z_{\text {center }}^{T}-z_{\text {center }}^{O}\right)^{2}},
$$

We have to take into account the fact that (4.33) presents idealistic case for two points in space, whereas for Target and Object from Figure 6 we have to use their real size values. For this purpose, given (4.4) and (4.5) we introduce the following.

$$
\operatorname{dim}^{T}=\max \left\{w^{T}, d^{T}, h^{T}\right\} ; \operatorname{dim}^{O}=\max \left\{w^{O}, d^{O}, h^{O}\right\} .
$$

Therefore, the real distance between Target and Object from Figure 6, given (4.33), (4.34) could be defined like that

$$
d_{\text {Real }}(T, O)=d(T, O)-\operatorname{dim}^{T} / 2-\operatorname{dim}^{O} / 2
$$

We define the degree $N_{\left(\operatorname{dim}^{T}, \operatorname{dim}^{O}\right)}(T, O)$, to which a Target and an Object, i.e., two points $T_{\text {center }}, O_{\text {center }} \in \mathfrak{R}^{3}$, given (4.34) and (4.25) are near each other, by using an idea from [9] as

$$
N_{\left(\operatorname{dim}^{T}, \operatorname{dim}^{O}\right)}(T, O)=\left\{\begin{array}{l}
1, d_{\text {Real }}(T, O) \leq \operatorname{dim}^{T}, \\
0, d_{\text {Real }}(T, O)>\operatorname{dim}^{T}+\operatorname{dim}^{O}, \\
\frac{\operatorname{dim}^{T}+\operatorname{dim}^{O}-d_{\text {Real }}(T, O)}{\operatorname{dim}^{O}}, \text { othervise. }
\end{array}\right.
$$

Note how $N_{\left(\operatorname{dim}^{T}, \operatorname{dim}^{O}\right)}(T, O)$ can be regarded as the degree to which the distance between a Target and an Object is at most about $\operatorname{dim}^{T}$. The value of $\operatorname{dim}^{O}$ defines how flexible "about $\operatorname{dim}^{T}$ " is interpreted. On the other hand, the degree $F_{\left(\operatorname{dim}^{T}, \operatorname{dim}^{O}\right)}(T, O)$ to which a Target and an Object, i.e., two points $T_{\text {center }}, O_{\text {center }} \in \mathfrak{R}^{3}$, given (4.34) and (4.25) are far from each other is defined like $F_{\left(\operatorname{dim}^{T}, \operatorname{dim}^{O}\right)}(T, O)=1-N_{\left(\operatorname{dim}^{T}, \operatorname{dim}^{O}\right)}(T, O)$, i.e.

$$
F_{\left(\operatorname{dim}^{T}, \operatorname{dim}^{O}\right)}(T, O)=\left\{\begin{array}{l}
0, d_{\text {Real }}(T, O) \leq \operatorname{dim}^{T}, \\
1, d_{\text {Real }}(T, O)>\operatorname{dim}^{T}+\operatorname{dim}^{O}, \\
\frac{d_{\text {Real }}(T, O)-\operatorname{dim}^{T}}{\operatorname{dim}^{O}}, \text { othervise. }
\end{array}\right.
$$

The relationship between $N_{\left(\operatorname{dim}^{T}, \operatorname{dim}^{O}\right)}(T, O)$ and $F_{\left(\operatorname{dim}^{T}, \operatorname{dim}^{O}\right)}(T, O)$ on one hand, and $d_{\text {Real }}(T, O)$ on the other is depicted in Figure 8.

We conclude this work with the last important fuzzy feature, related to a human like perception of a world by a set of terms like "North-East-South-West", when each of sub terms could be represented by an angle $\theta(x, y)$ from (4.19). Using a technique from [3] we model a vague cardinal direction by three parameters: $\theta, \operatorname{dev} \theta$ and $\Delta \theta$, where $\theta$ is the most prototypical angle for each cardinal direction, i.e. "North", "East", "South" or "West" and the allowed deviation 
(a)

1

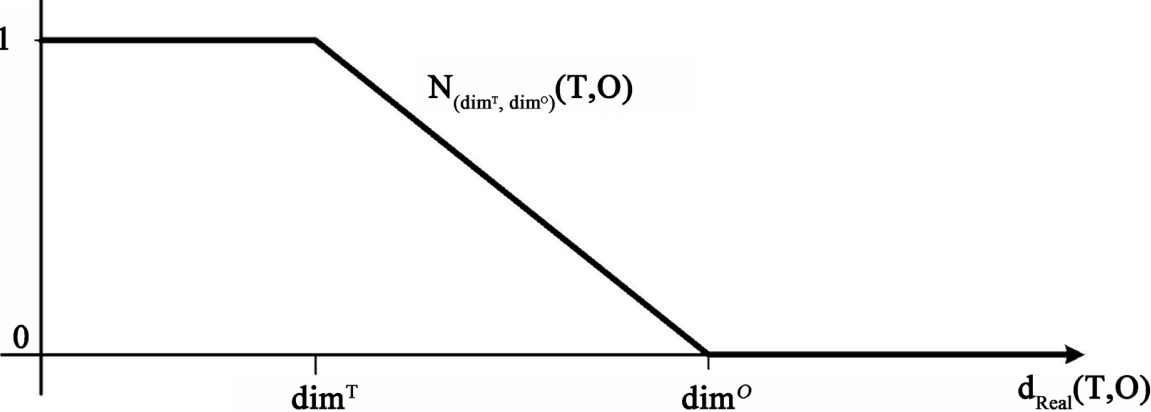

(b)

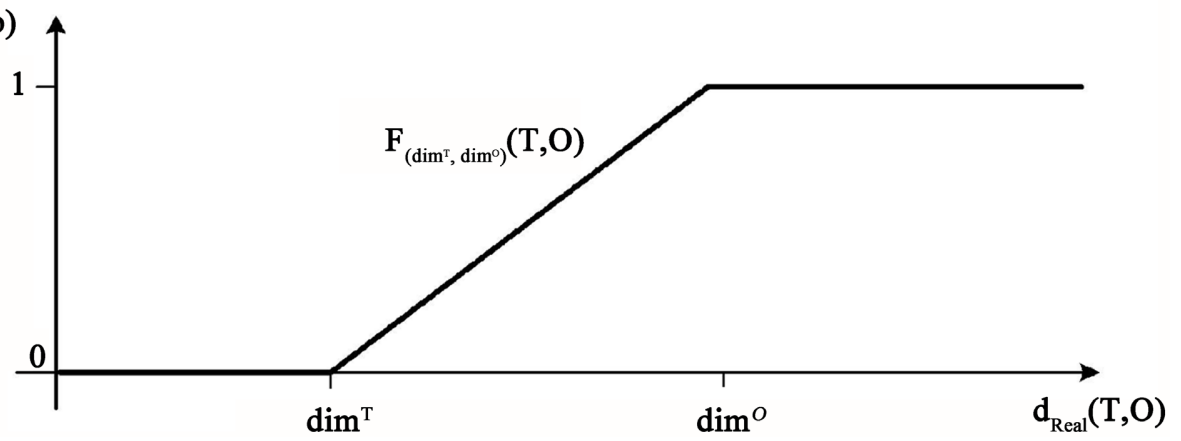

Figure 8. Relationship between the degree to which points $T_{\text {center }}, O_{\text {center }} \in \mathfrak{R}^{3}$ are near (a) or far (b) from each other, and their distance $d_{\text {Real }}(T, O)$.

from $\theta$ is about $\operatorname{dev} \theta$. Again $\Delta \theta$ models how flexible "about $\operatorname{dev} \theta$ " is interpreted. We define $D_{(\theta(x, y), \operatorname{dev} \theta, \Delta \theta)}(T, O)$ as

$$
D_{(\theta(x, y), \operatorname{dev} \theta, \Delta \theta)}(T, O)=\left\{\begin{array}{l}
1, \operatorname{ad}(\theta(x, y), \theta) \leq \operatorname{dev} \theta, \\
0, \operatorname{ad}(\theta(x, y), \theta)>\operatorname{dev} \theta+\Delta \theta, \\
\frac{\operatorname{dev} \theta+\Delta \theta-\operatorname{ad}(\theta(x, y), \theta)}{\Delta \theta}, \text { othervise. }
\end{array}\right.
$$

where $\operatorname{ad}(\ldots)$ represents the unsigned angular difference [3], i.e., for $\theta_{1}$ and $\theta_{2}$ in $\mathrm{R}$, we define

$$
\operatorname{ad}\left(\theta_{1}, \theta_{2}\right)=\min \left(\operatorname{norm}\left(\theta_{2}-\theta_{1}\right), 2 \pi-\operatorname{norm}\left(\theta_{2}-\theta_{1}\right)\right),
$$

where $\operatorname{norm}(\theta)=\theta+2 k \pi$ and $k$ is the unique integer satisfying $\theta+2 k \pi \in[0,2 \pi]$. By analogy, we define the positive angular difference pad for $\theta_{1}$ and $\theta_{2}$ in $\Re$ by

$$
\operatorname{pad}\left(\theta_{1}, \theta_{2}\right)=\operatorname{norm}\left(\theta_{2}-\theta_{1}\right) \text {. }
$$

For example, "East" could be modelled by the fuzzy relation $D_{\left(\frac{\pi}{2}, \frac{\pi}{8}, \frac{\pi}{4}\right)}$. The fuzzy set of points, which are "East" of a reference point $T_{\text {center }}$, using this interpretation, is displayed in Figure 9. In this figure, membership degrees $D_{\left(\frac{\pi}{2}, \frac{\pi}{8}, \frac{\pi}{4}\right)}(T, O)$ for various points $O_{\text {center }}$ are depicted using grayscale colors, black being membership degree 1 and white being 0 .

Now we are going to put together a set of an elements of Object/Target mutual 


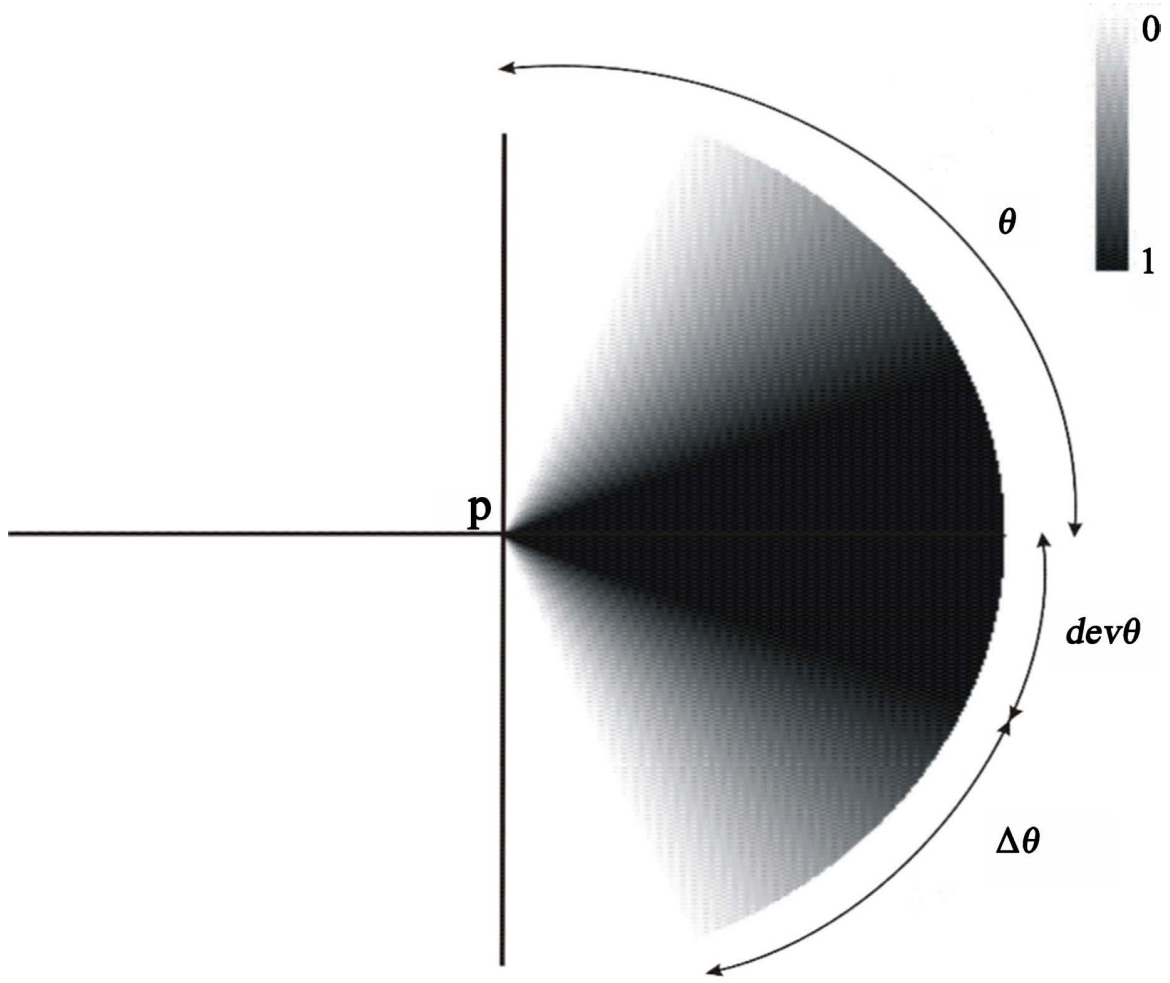

Figure 9. Fuzzy set $D_{\left(\frac{\pi}{2}, \frac{\pi}{8}, \frac{\pi}{4}\right)}(p,$.$) of points which are east of p$.

behavior. Let us formulate the "Tactical Level" of an Object decision making mechanism, the goal of which is to figure out if there is a way to achieve the ultimate outcome of its potential ACTIONs.

\subsection{Feasibility of a Goal Setting}

Let us define a notion of a Pre-conditions and GOALs of an Object and a Target mutual interaction. From the position of common sense, we consider a set of Size Comparison Predicates from (4.6), (4.7), Mutual Positioning Predicates from (4.8)-(4.15) and Entity Shape Estimation Predicates from (4.23)-(4.26) as a set of Pre-conditions to find out if any particular GOAL of an Object/Target setting is achievable. We also consider the set of Docking Positioning Predicates from (4.27)-(4.32) as an Object action GOALs (for the sake of current discussion).

We have to note that by the term Docking we presume unfriendly (no specific docking mechanism in place) approaching of a Target by an Object. We also presume that the size of an Object is $S M A L L E R$, than a Target one. It means that both (4.38) and (4.39) as well, as all sentences from an APPENDIX, should also include $T(\operatorname{SMALLER}(O$, Tar $))$ term.

A feasibility of achieving a Target by an Object could be described by the set of predicates based logical sentences of the following structure.

$T(R G F(O)) \wedge T(R G F($ Tar $)) \wedge T(\operatorname{HIGHER}(O$, Tar $)) \Rightarrow T(D O T(O, T a r))$

This (4.38) logical sentence formulates a specific rule, which could be pre- 
sented by natural language as follows: "IF both a Target and an Object have right geometric form AND an Object is positioned HIGHER, than a Target AND Object's size is $S M A L L E R$, THEN the GOAL of an Object being docked on TOP of a Target is achievable.

Similarly, the following sentence formulates an opposite logical outcome.

$T(R G F(O)) \wedge T(R G F($ Tar $)) \wedge T(\operatorname{LOWER}(O$, Tar $)) \Rightarrow F(D O T(O, T a r))(4.39)$

This (4.39) logical sentence formulates a specific rule, which could be presented in natural language as follows: "IF both a Target and an Object have right geometric form AND an Object is positioned LOWER, than a Target AND Object's size is $S M A L L E R$, than Target's one, THEN the GOAL of an Object being docked on $T O P$ of a Target is NOT achievable.

Let us also consider a couple cases from an APPENDIX, which describe another logical presumption.

$$
T(R G F(O)) \wedge T(R G F(\text { Tar })) \wedge T(\operatorname{ATLEFT}(O, \text { Tar })) \Rightarrow T(D A L(O, T a r))
$$

This (4.40) logical sentence formulates a specific rule, which could be presented by natural language as follows: "IF both a Target and an Object have right geometric form AND an Object is positioned ATLEFT of a Target AND Object's size is SMALLER, THEN the GOAL of an Object being docked at LEFT of a Target is achievable.

$T(R G F(O)) \wedge T(R G F($ Tar $)) \wedge T($ ATRIGHT $(O$, Tar $)) \Rightarrow F(D A L(O$, Tar $))$

This (4.41) logical sentence formulates a specific rule, which could be presented by natural language as follows: "IF both a Target and an Object have right geometric form AND an Object is positioned ATRIGHT of a Target AND Object's size is SMALLER, THEN the GOAL of an Object being docked at LEFT of a Target is NOT achievable.

We have to point, that some sentences from an APPENDIX have a form of a prohibition. For instance, when the Entity has flat left and right surfaces (FLRS), then the following is taking place

$$
T(\operatorname{FLRS}(O)) \wedge T(F L R S(\text { Tar })) \Rightarrow F(\text { DOT }(O, \text { Tar }))
$$

This (4.42) logical sentence formulates a specific rule, which could be presented by natural language as follows: "IF both a Target and an Object have flat left and right surfaces, THEN the GOAL of an Object being docked on TOP of a Target is NOT achievable. The same logic (prohibition) is applied for an OBJECT for being at the BOTTOM, FRONT or BACK of a Target.

Let's underscore the fact, that (4.38)-(4.42) represent feasibility of a goal settings in its Boolean form and, of course, could not be always applied to a real-world situation. Therefore, we shall try to introduce its fuzzy counterpart.

\subsection{Fuzzification of Feasibility of a Goal Setting}

We are not pretending to generalize the way to re-interpret Size Comparison Predicates from (4.6), (4.7), Mutual Positioning Predicates from (4.8)-(4.15) and 
Entity Shape Estimation Predicates from (4.23)-(4.26), as well as Docking Positioning Predicates from (4.27)-(4.32) in fuzzy way, but still try to apply a certain uniform approach, extensively used in [5] and [6]. For this purpose, we are normalizing

1) Vertical (Z-dimension) derivation from (4.20)

$$
\begin{aligned}
& \forall x, y \mid x \in\left[x_{\min }, x_{\max }\right], y \in\left[y_{\min }, y_{\max }\right], \forall i \in[1, N], \forall j \in[1, M] \\
& \Rightarrow \Delta z\left(x_{i}, y_{j}\right)^{\text {norm }}=\frac{z\left(x_{i}, y_{j}\right)-z^{\min }(x, y)}{z^{\max }(x, y)-z^{\min }(x, y)} .
\end{aligned}
$$

Note, that from (4.43) we can calculate the following

$$
\begin{gathered}
\Delta z(x, y)^{\text {norm }}=\frac{\sum_{N \times M} \Delta z\left(x_{i}, y_{j}\right)^{\text {norm }}}{N \times M}, \\
\text { where } \Delta z(x, y)^{\text {norm }} \in[0,1] .
\end{gathered}
$$

To define if an object/target has Right Geometrical Form on Vertical (Z-dimension) we propose the following

$$
\Delta z(x, y)=\frac{\left|\Delta z(x, y)^{\text {norm }}-0.5\right|}{0.5},
$$

In (4.46) the value of 0.5 means that there are a lot of different "tops" and "bottoms" of $\Delta z(x, y)^{\text {norm }}$ on a vertical surface of an entity, which effectively resulted in $\Delta z(x, y)=0$. Otherwise, when $\Delta z(x, y)^{\text {norm }} \approx 0$ or $\Delta z(x, y)^{\text {norm }} \approx 0$, we could consider a vertical surface as geometrically perfect one (flat).

We represent $\Delta z(x, y)$ from (4.46) as a fuzzy set, forming linguistic variable, described by a triplet of the form $\Delta Z=\left\{\left\langle\Delta Z_{i}, U_{z}, \Delta \tilde{Z}\right\rangle\right\}, \Delta Z_{i} \in T\left(u_{z}\right)$, $\forall i \in\left[0, \operatorname{Card}_{z}\right]$, where $T_{i}(u)$ is extended term set of the linguistic variable "Vertical derivation "from Table $5, \tilde{Z}$ is normal fuzzy set with correspondent membership function $\mu_{\Delta z}: U_{z} \rightarrow[0,1]$.

We will use the following mapping

$$
\begin{gathered}
\alpha: \widetilde{\Delta \tilde{Z}} \rightarrow U_{z}\left|u_{i}=\operatorname{Ent}\left[\left(\operatorname{Card}_{z}-1\right) \times \Delta Z_{i}\right]\right| \forall i \in\left[0, \operatorname{CardU}_{z}\right] \text {, were } \\
\widetilde{\Delta Z}=\int_{U_{z}} \mu_{\Delta z}\left(u_{z}\right) / u_{z}
\end{gathered}
$$

On the other hand, similarly to the previous cases, to determine the estimates of the membership function in terms of singletons from (4.46) in the form $\mu_{\Delta z_{i}}\left(\Delta z_{i}\right) / \Delta z_{i} \mid \forall i \in\left[0, \operatorname{Card}_{z}\right]$ we propose the following procedure.

$\forall i \in\left[0, \operatorname{Card}_{z}\right], \mu_{\Delta z_{i}}\left(\Delta z_{i}\right)=1-\frac{1}{\operatorname{Card}_{z}-1} \times\left|i-\operatorname{Ent}\left[\left(\operatorname{Card}_{z}-1\right) \times \Delta Z_{i}\right]\right|$

2) Horizontal (X-dimension) derivation from (4.21)

$$
\begin{aligned}
& \forall z, y \mid z \in\left[z_{\min }, z_{\max }\right], y \in\left[y_{\min }, y_{\max }\right], \forall k \in[1, L], \forall j \in[1, M] \\
& \Rightarrow \Delta x\left(z_{k}, y_{j}\right)^{\text {norm }}=\frac{x\left(z_{k}, y_{j}\right)-x^{\min }(z, y)}{x^{\max }(z, y)-x^{\min }(z, y)} .
\end{aligned}
$$

Note, that from (4.49) we can calculate the following 


$$
\begin{gathered}
\Delta x(z, y)^{\text {norm }}=\frac{\sum_{L \times M} \Delta x\left(z_{k}, y_{j}\right)^{\text {norm }}}{L \times M}, \\
\text { where } \Delta x(z, y)^{\text {norm }} \in[0,1] .
\end{gathered}
$$

To define if an object/target has Right Geometrical Form on Horizontal (X-dimension) we propose the following

$$
\Delta x(z, y)=\frac{\left|\Delta x(z, y)^{\text {norm }}-0.5\right|}{0.5},
$$

Once again, we represent $\Delta x(z, y)$ from (4.52) as a fuzzy set, forming linguistic variable, described by a triplet of the form $\Delta X=\left\{\left\langle\Delta X_{i}, U_{X}, \Delta \tilde{X}\right\rangle\right\}$, $\Delta X_{i} \in T\left(u_{x}\right), \forall i \in\left[0, \operatorname{Card}_{x}\right]$, where $T_{i}(u)$ is extended term set of the linguistic variable "Horizontal derivation "from Table 5, $\tilde{X}$ is normal fuzzy set with correspondent membership function $\mu_{\Delta x}: U_{x} \rightarrow[0,1]$.

We will use the following mapping

$$
\begin{gathered}
\beta: \widetilde{\Delta \widetilde{X}} \rightarrow U_{x}\left|u_{i}=\operatorname{Ent}\left[\left(\operatorname{Card}_{x}-1\right) \times \Delta X_{i}\right]\right| \forall i \in\left[0, \operatorname{Card}_{x}\right] \text {, were } \\
\widetilde{\Delta X}=\int_{U_{x}} \mu_{\Delta x}\left(u_{x}\right) / u_{x} .
\end{gathered}
$$

On the other hand, similarly to the previous cases, to determine the estimates of the membership function in terms of singletons from (4.51) in the form $\mu_{\Delta x_{i}}\left(\Delta x_{i}\right) / \Delta x_{i} \mid \forall i \in\left[0, \operatorname{Card}_{x}\right]$ we propose the following procedure.

$\forall i \in\left[0, \operatorname{Card}_{x}\right], \mu_{\Delta x_{i}}\left(\Delta x_{i}\right)=1-\frac{1}{\operatorname{Card}_{x}-1} \times\left|i-\operatorname{Ent}\left[\left(\operatorname{Card}_{x}-1\right) \times \Delta X_{i}\right]\right|$

3) Y-dimension derivation from (4.22)

$$
\begin{aligned}
& \forall x, z \mid x \in\left[x_{\min }, x_{\max }\right], z \in\left[z_{\min }, z_{\text {max }}\right], \forall i \in[1, N], \forall k \in[1, L] \\
& \Rightarrow \Delta y\left(x_{i}, z_{k}\right)^{n o r m}=\frac{y\left(x_{i}, z_{k}\right)-y^{\min }(x, z)}{y^{\max }(x, z)-y^{\min }(x, z)} .
\end{aligned}
$$

Note, that from (4.49) we can calculate the following

$$
\begin{gathered}
\Delta y(x, z)^{\text {norm }}=\frac{\sum_{N \times L} \Delta y\left(x_{i}, z_{k}\right)^{\text {norm }}}{N \times L}, \\
\text { where } \Delta y(x, z)^{\text {norm }} \in[0,1] .
\end{gathered}
$$

To define if an object/target has Right Geometrical Form on Y-dimension we propose the following

$$
\Delta y(x, z)=\frac{\left|\Delta y(x, z)^{\text {norm }}-0.5\right|}{0.5}
$$

Once again, we represent $\Delta y(x, z)$ from (4.58) as a fuzzy set, forming linguistic variable, described by a triplet of the form $\Delta Y=\left\{\left\langle\Delta Y_{i}, U_{Y}, \Delta \tilde{Y}\right\rangle\right\}$, $\Delta Y_{i} \in T\left(u_{y}\right), \forall i \in\left[0, \operatorname{Card}_{y}\right]$, where $T_{i}(u)$ is extended term set of the linguistic variable " $Y$-dimension derivation "from Table 5, $\tilde{X}$ is normal fuzzy set with correspondent membership function $\mu_{\Delta y}: U_{y} \rightarrow[0,1]$. 
Table 5. Linguistic variables for dimensions derivation and geometric form.

\begin{tabular}{|c|c|c|}
\hline \multicolumn{2}{|c|}{ Value of variable } & $\Delta z_{i} \in U_{z}, \Delta x_{i} \in U_{x}, \Delta y_{i} \in U_{y}$, \\
\hline $\begin{array}{l}\text { "Vertical/Horizontal/ } \\
Y \text {-dimension derivation" }\end{array}$ & $\begin{array}{l}\text { Right geometric } \\
\text { form }\end{array}$ & $r g f_{i} \in U_{R G F} \quad \forall i \in[0,4]$ \\
\hline ideal surface & perfect & 0 \\
\hline very low & acceptable & 1 \\
\hline low & in doubt & 2 \\
\hline medium & far from right & 3 \\
\hline high & not right at all & 4 \\
\hline
\end{tabular}

We will use the following mapping

$$
\begin{gathered}
\Omega: \widetilde{\Delta \tilde{Y}} \rightarrow U_{y}\left|u_{i}=\operatorname{Ent}\left[\left(\operatorname{CardU}_{y}-1\right) \times \Delta Y_{i}\right]\right| \forall i \in\left[0, \operatorname{CardU}_{y}\right] \text {, were } \\
\widetilde{\Delta Y}=\int_{U_{y}} \mu_{\Delta y}\left(u_{y}\right) / u_{y} .
\end{gathered}
$$

On the other hand, similarly to the previous cases, to determine the estimates of the membership function in terms of singletons from (4.5) in the form $\mu_{\Delta y_{i}}\left(\Delta y_{i}\right) / \Delta y_{i} \mid \forall i \in\left[0, \operatorname{Card}_{y}\right]$ we propose the following procedure.

$\forall i \in\left[0, \operatorname{Card}_{y}\right], \mu_{\Delta y_{i}}\left(\Delta y_{i}\right)=1-\frac{1}{\operatorname{Card}_{y}-1} \times\left|i-\operatorname{Ent}\left[\left(\operatorname{Card}_{y}-1\right) \times \Delta Y_{i}\right]\right|$

Note that from (4.23)-(4.26) we have the following predicate based logical statement

$$
T(F L R S(E)) \wedge T(F T B(E)) \wedge T(F F B S(E)) \Rightarrow T(R G F(E)),
$$

Which means that the right geometric form of an entity depends on Vertical/Horizontal/ $Y$-dimension derivations, therefore we represent $R G F$ from (4.61) as a fuzzy set, forming linguistic variable, described by a triplet of the form $R G F=\left\{\left\langle R G F_{i}, U_{R G F}, \overparen{R G F}\right\rangle\right\}, \quad R G F_{i} \in T\left(u_{r g f}\right), \quad \forall i \in\left[0, \operatorname{Card}_{R G F}\right]$, where $T_{i}(u)$ is extended term set of the linguistic variable "right geometric form" from Table 5, $\widetilde{R G F}$ is normal fuzzy set with correspondent membership function $\mu_{r g f}: U_{R D F} \rightarrow[0,1]$. We will use the following mapping

$$
\begin{gathered}
\alpha: \overparen{R G F} \rightarrow U_{R G F}\left|u_{i}=\operatorname{Ent}\left[\left(\operatorname{Card}_{R G F}-1\right) \times R G F_{i}\right]\right| \forall i \in\left[0, \operatorname{Card}_{R G F}\right] \text {, i.e. } \\
\widetilde{R G F}=\int_{U_{R G F}} \mu_{R G F}\left(u_{r g f}\right) / u_{r g f} .
\end{gathered}
$$

On the other hand, similarly to the previous cases, to determine the estimates of the membership function in terms of singletons from (4.5) in the form $\mu_{r g f_{i}}\left(r g f_{i}\right) / r g f_{i} \mid \forall i \in\left[0, \operatorname{CardU}_{R G F}\right]$ we propose the following procedure.

$$
\begin{aligned}
& \forall i \in\left[0, \operatorname{Card}_{R G F}\right], \\
& \mu_{r g f_{i}}\left(r g f_{i}\right)=1-\frac{1}{\operatorname{Card}_{R G F}-1} \times\left|i-\operatorname{Ent}\left[\left(\operatorname{Card}_{R G F}-1\right) \times R G F_{i}\right]\right|
\end{aligned}
$$

To convert (4.61) into fuzzy logic-based statement and terms from Table 5 we use a Fuzzy Conditional Inference Rule, formulated by means of "common 
sense" as a following conditional clause:

$P=$ "IF ( $\widetilde{\Delta Z}$ is $Z$ ) $\operatorname{AND}(\widetilde{\Delta X}$ is $X) \operatorname{AND}(\widetilde{\Delta Y}$ is $Y)$, THEN ( $\widetilde{R G F}$ is $R G F)$ " (4.64)

In other words, we use fuzzy conditional inference of the following type [7] [8]:

Ant 1: If $\Delta z$ is $Z$ and $\Delta x$ is $X$ and $\Delta y$ is $Y$ then $r g f$ is $R G F$

Ant 2: $\Delta z$ is $Z^{\prime}$ and $\Delta x$ is $X^{\prime}$ and $\Delta y$ is $Y^{\prime}$

Cons: $r g f$ is $R G F^{\prime}$

where $Z, Z^{\prime} \subseteq U_{Z}, \quad X, X^{\prime} \subseteq U_{X}, Y, Y^{\prime} \subseteq U_{Y}$ and $R G F, R G F^{\prime} \subseteq U_{R G F}$.

Now for fuzzy sets (4.47), (4.53), (4.59) and (4.62) a binary relationship for the fuzzy conditional proposition of the type (4.64) and (4.65) for fuzzy logic we use so far is defined as

$$
\begin{aligned}
& R\left(A_{1}(z, x, y), A_{2}(r g f)\right)=\left[Z \times U_{Z} \cap X \times U_{X} \cap Y \times U_{Y}\right] \\
& \rightarrow R G F \times U_{R G F}=\int_{U_{Z} \times U_{X} \times U_{Y} \times U_{R G F}}\left(\left[\mu_{\Delta z}\left(u_{z}\right) / u_{z} \wedge \mu_{\Delta x}\left(u_{x}\right) / u_{x} \wedge \mu_{\Delta y}\left(u_{y}\right) / u_{y}\right]\right. \\
& \left.\rightarrow \mu_{R G F}\left(u_{r g f}\right) / u_{r g f}\right) /\left(u_{z}, u_{x}, u_{y}, u_{r g f}\right)
\end{aligned}
$$

Given (2.10) and since we consider that

$\operatorname{Card}_{Z}=\operatorname{Card}_{X}=\operatorname{Card}_{Y}=\operatorname{Card}_{R G F}$, then expression (4.66) looks like

$$
\begin{gathered}
{\left[\mu_{\Delta z}\left(u_{z}\right) / u_{z} \wedge \mu_{\Delta x}\left(u_{x}\right) / u_{x} \wedge \mu_{\Delta y}\left(u_{y}\right) / u_{y}\right] \rightarrow \mu_{R G F}\left(u_{r g f}\right) / u_{r g f}} \\
=\left\{\begin{array}{c}
\left(1-\left[\mu_{\Delta z}\left(u_{z}\right) \wedge \mu_{\Delta x}\left(u_{x}\right) \wedge \mu_{\Delta y}\left(u_{y}\right)\right] \cdot \mu_{R G F}\left(u_{r g f}\right),\left[\mu_{\Delta z}\left(u_{z}\right) \wedge \mu_{\Delta x}\left(u_{x}\right) \wedge \mu_{\Delta y}\left(u_{y}\right)\right]>\mu_{R G F}\left(u_{r g f}\right),\right. \text { (4.67) } \\
1,\left[\mu_{\Delta z}\left(u_{z}\right) \wedge \mu_{\Delta x}\left(u_{x}\right) \wedge \mu_{\Delta y}\left(u_{y}\right)\right] \leq \mu_{R G F}\left(u_{r g f}\right) .
\end{array}\right. \\
\text { where }\left[\mu_{\Delta z}\left(u_{z}\right) \wedge \mu_{\Delta x}\left(u_{x}\right) \wedge \mu_{\Delta y}\left(u_{y}\right)\right] \text { is min }\left[\mu_{\Delta z}\left(u_{z}\right), \mu_{\Delta x}\left(u_{x}\right), \mu_{\Delta y}\left(u_{y}\right)\right] \text {. It is } \\
\text { well known that given a unary relationship } R\left(A_{1}\left(z^{\prime}, x^{\prime}, y^{\prime}\right)\right)=Z^{\prime} \cap X^{\prime} \cap Y^{\prime} \text { one } \\
\text { can obtain the consequence } R\left(A_{2}(r g f)\right) \text { by applying compositional rule of in- } \\
\text { ference }(\mathrm{CRI}) \text { to } R\left(A_{1}\left(z^{\prime}, x^{\prime}, y^{\prime}\right)\right) \text { and } R\left(A_{1}(z, x, y), A_{2}(r g f)\right) \text { of type (4.66): } \\
R\left(A_{2}(r g f)\right)=Z^{\prime} \cap X^{\prime} \cap Y^{\prime} \circ R\left(A_{1}(z, x, y), A_{2}(r g f)\right) \\
=\int_{U_{Z} \times U_{X} \times U_{Y}}\left[\mu_{\Delta z^{\prime}}\left(u_{z}\right) \wedge \mu_{\Delta x^{\prime}}\left(u_{x}\right) \wedge \mu_{\Delta y^{\prime}}\left(u_{y}\right)\right] /\left(u_{z}, u_{x}, u_{y}\right) \\
\circ \int_{U_{Z} \times U_{X} \times U_{Y} \times U_{R G F}}\left[\mu_{\Delta z}\left(u_{z}\right) \wedge \mu_{\Delta x}\left(u_{x}\right) \wedge \mu_{\Delta y}\left(u_{y}\right)\right] \rightarrow \mu_{R G F}\left(u_{r g f}\right) /\left(u_{z}, u_{x}, u_{y}, u_{r g f}\right) \text { (4.68) } \\
=\int_{R G F} \bigcup_{z \in U_{z}, y \in U_{y}, x \in U_{x}}\left\{\left[\mu_{\Delta z^{\prime}}\left(u_{z}\right) \wedge \mu_{\Delta x^{\prime}}\left(u_{x}\right) \wedge \mu_{\Delta y^{\prime}}\left(u_{y}\right)\right]\right. \\
\left.\wedge\left(\left[\mu_{\Delta z}\left(u_{z}\right) \wedge \mu_{\Delta x}\left(u_{x}\right) \wedge \mu_{\Delta y}\left(u_{y}\right)\right] \rightarrow \mu_{R G F}\left(u_{r g f}\right)\right)\right\} / u_{r g f}
\end{gathered}
$$

But for practical purposes we will use another Fuzzy Conditional Rule (FCR)

$$
\begin{aligned}
& R\left(A_{1}(z, x, y), A_{2}(\text { rgf })\right)=(P \times U \rightarrow V \times S) \cap(\neg P \times U \rightarrow V \times \neg S) \\
& =\int_{U \times V}\left(\mu_{P}(u) \rightarrow \mu_{S}(v)\right) \wedge\left(\left(1-\mu_{P}(u)\right) \rightarrow\left(1-\mu_{S}(v)\right)\right) /(u, v)
\end{aligned}
$$

where $P=Z \cap X \cap Y$ and $U=U_{Z}=U_{X}=U_{Y}$, therefore from (4.69) we are getting 


$$
\begin{aligned}
R\left(A_{1}(z, x, y), A_{2}(r g f)\right)= & \left(\mu_{P}(u) \rightarrow \mu_{S}(v)\right) \wedge\left(\left(1-\mu_{P}(u)\right) \rightarrow\left(1-\mu_{S}(v)\right)\right) \\
= & \left\{\begin{array}{l}
\left(1-\mu_{P}(u)\right) \cdot \mu_{S}(v), \mu_{P}(u)>\mu_{S}(v), \\
1, \mu_{P}(u)=\mu_{S}(v), \\
\left(1-\mu_{S}(v)\right) \cdot \mu_{P}(u), \mu_{P}(u)<\mu_{S}(v) .
\end{array}\right.
\end{aligned}
$$

As was already mentioned above, $F C R$ from (4.70) gives more reliable results.

\subsection{Example}

To build a binary relationship matrix of type (4.69) we us use a conditional clause of type (4.64):

$P=$ "IF ( $Z$ is "ideal surface") AND ( $X$ is "ideal surface") AND ( $Y$ is "ideal surface"), THEN ( $R G F$ is "perfect")"

To build membership functions for fuzzy sets $Z, X$ and $Y$ we use (4.47), (4.53) and (4.59) respectively.

In (4.47) the membership functions for fuzzy set $Z$ (for instance from Table 5) would look like:

$$
\begin{aligned}
\mu_{P}(u) & =\mu_{Z}(\text { "ideal surface" }) \wedge \mu_{X}(\text { "ideal surface" }) \wedge \mu_{Y}(\text { "ideal surface" }) \\
& =1 / 0+0.75 / 1+0.5 / 2+0.25 / 3+0 / 4
\end{aligned}
$$

Same membership functions we use for fuzzy sets $X$ and $Y$. Note, that the membership function for fuzzy set $R G F$ from Table 5 is also the same

$$
\mu_{R G F} \text { ("perfect") }=1 / 0+0.75 / 1+0.5 / 2+0.25 / 3+0 / 4
$$

Given (4.70), (4.72) and (4.73) we have $R\left(A_{1}(z, x, y), A_{2}(r g f)\right)$ shown in Table 6.

Suppose that the current value of "horizontal derivation", represented by a fuzzy set $X^{\prime}$ from (4.53), is defined as

$\mu_{X^{\prime}}$ ("high" $)=0 / 0+0.25 / 1+0.5 / 2+0.75 / 3+1 / 4$ and ( $Z^{\prime}$ is “ideal surface") AND ( $Y^{\prime}$ is 'ideal surface') and

$$
\begin{aligned}
\mu_{P^{\prime}}(u) & \left.=\mu_{Z^{\prime}}(\text { "ideal surface" }) \wedge \mu_{X^{\prime}}(\text { "high" }) \wedge \mu_{Y^{\prime}} \text { ("ideal surface" }\right) . \\
& =0 / 0+0.25 / 1+0.5 / 2+0.25 / 3+0 / 4
\end{aligned}
$$

After applying CRI from (4.68) we get the following $R\left(A_{2}(r g f)\right)=0 / 0+0.25 / 1+0.5 / 2+0.25 / 3+0 / 4$ and after membership function normalization we are getting $\mu_{R G F^{\prime}}$ ("in doubt") $=0 / 0+0.5 / 1+1 / 2+0.5 / 3+0 / 4$,

Table 6. Binary relationship matrix of a current example.

\begin{tabular}{cccccc}
\hline$p \rightarrow r g f$ & $\mathbf{1}$ & $\mathbf{0 . 7 5}$ & $\mathbf{0 . 5}$ & $\mathbf{0 . 2 5}$ & $\mathbf{0}$ \\
\hline 1 & 1 & 0 & 0 & 0 & 0 \\
0.75 & 0 & 1 & 0.125 & 0.0625 & 0 \\
0.5 & 0 & 0.125 & 1 & 0.125 & 0 \\
0.25 & 0 & 0.0625 & 0.125 & 1 & 0 \\
0 & 0 & 0 & 0 & 0 & 1 \\
\hline
\end{tabular}


which means (4.61) is not fully satisfactory and an Entity has to re-orient itself to successfully "dock" to another one.

All elements of a predicates based logical sentences ((4.38), for instance) like structure such as "FLRS“, "FTB“, "FFBS“, "SMALLER", "LARGER", "HIGHER", "LOWER" etc. from an Appendix, could be presented by above-described technique.

The summary of this presentation:

1) The AIA strategic targeting could be based on an axiomatic geometry and extended objects representation.

2) The AIA intermediary behavior trigger could be based on both $(4,36)$, (4.37) between entities fuzzy distance and "Noth-East-South-West" orientation estimates.

3) The AIA tactical behavior could be defined by fuzzification of an element of a predicate based logical sentences, very limited subset of which are presented in an APPENDIX.

\section{Conclusion}

In this work it was shown that the AIA strategic targeting could be based on approximate geometric behavior of extended objects, described by fuzzy predicates. The axiom system of Boolean Euclidean geometry was fuzzified and formalized in the language of fuzzy logic, presented in [1]. Based on the same fuzzy logic, we formulated a special form of positional uncertainty, namely positional tolerance that arises from geometric constructions with extended primitives. We also addressed Euclid's first postulate, which lays the foundation for consistent geometric reasoning in all classical geometries by considered extended primitives and gave a fuzzification of Euclid's first postulate by using the same fuzzy logic. Fuzzy equivalence relation "Extended lines sameness" is introduced. We also use the fuzzy logic from [1] for fuzzy conditional inference determination, elements of which were "Degree of indiscernibility" and "Discernibility measure "of extended points. We also presented some logical principles of AIA orientation, which will allow an implementation of its fuzzy "tactical" decision making.

\section{Conflicts of Interest}

The author declares no conflicts of interest regarding the publication of this paper.

\section{References}

[1] Tserkovny, A. (2017) A t-Norm Fuzzy Logic for Approximate Reasoning. Journal of Software Engineering and Applications, 10, 639-661. https://doi.org/10.4236/jsea.2017.107035

[2] Genesereth, M. and Nilsson, N.J. (1989) Logical Foundations of Artificial Intelligence. Morgan Kaufmann Publishers, Los Altos. https://doi.org/10.1016/0004-3702(89)90073-8

[3] Tserkovny, A. (2016) Fuzzy Logic for Incidence Geometry. The Scientific World 
Journal, 2016, Article ID 9057262. https://doi.org/10.1155/2016/9057263

[4] Wilke, G. (2014) Equality in Approximate Tolerance Geometry. Proceedings of the 7 th IEEE International Conference Intelligent Systems (IS 14), Springer International, Warsaw, 365-376. https://doi.org/10.1007/978-3-319-11313-5 33

[5] Aliev, R.A. and Tserkovny, A. (1988) The Knowledge Representation in Intelligent Robots Based on Fuzzy Sets. Doklady Mathematics, 37, 541-544.

[6] Aliev, R.A., Mamedova, G.A. and Tserkovny, A.E. (1991) Fuzzy Control Systems. Energoatomizdat, Moscow.

[7] Fukami, S., Mizumoto, M. and Tanaka, K. (1980) Some Considerations of Fuzzy Conditional Inference. Fuzzy Sets and Systems, 4, 243-273. https://doi.org/10.1016/0165-0114(80)90014-7

[8] Aliev, R.A. and Tserkovny, A. (2011) Systemic Approach to Fuzzy Logic Formalization for Approximate Reasoning. Information Sciences, 181, 1045-1059. https://doi.org/10.1016/j.ins.2010.11.021

[9] Schockaert, S., De Cock, M. and Kerre, E.E. (2008) Modelling Nearness and Cardinal Directions between Fuzzy Regions. Proceedings of 2008 IEEE 16th International Conference on Fuzzy Systems (FUZZ-IEEE), Hong Kong, 1-6 June 2008, 1548-1555. 


\section{Appendix}

$$
\begin{aligned}
& T(R G F(O)) \wedge T(R G F(\text { Tar })) \wedge T(\operatorname{SMALLER}(O, \text { Tar })) \Rightarrow T(\operatorname{DIF}(O, \text { Tar })) \\
& T(R G F(O)) \wedge T(R G F(\text { Tar })) \wedge T(\operatorname{SMALLER}(O, \text { Tar })) \Rightarrow T(D A B(O, T a r)) \\
& T(R G F(O)) \wedge T(R G F(\text { Tar })) \wedge T(\operatorname{SMALLER}(O, \text { Tar })) \Rightarrow T(D A L(O, T a r)) \\
& T(R G F(O)) \wedge T(R G F(\text { Tar })) \wedge T(\operatorname{SMALLER}(O, \text { Tar })) \Rightarrow T(D A R(O, T a r)) \\
& T(R G F(O)) \wedge T(R G F(\text { Tar })) \wedge T(\operatorname{SMALLER}(O, \text { Tar })) \Rightarrow T(\text { DOT }(\text { O,Tar })) \\
& T(R G F(O)) \wedge T(R G F(\operatorname{Tar})) \wedge T(\operatorname{ATLEFT}(O, \operatorname{Tar})) \Rightarrow T(D A L(O, \text { Tar })) \\
& T(R G F(O)) \wedge T(R G F(\text { Tar })) \wedge T(\text { ATRIGHT }(O, \text { Tar })) \Rightarrow T(D A R(O, \text { Tar })) \\
& T(R G F(O)) \wedge T(R G F(T a r)) \wedge T(\operatorname{ATLEFT}(O, T a r)) \Rightarrow F(D A R(O, T a r)) \\
& T(R G F(O)) \wedge T(R G F(\text { Tar })) \wedge T(\operatorname{ATRIGHT}(O, T a r)) \Rightarrow F(D A L(O, T a r)) \\
& T(\operatorname{FLRS}(O)) \wedge T(\operatorname{FLRS}(\text { Tar })) \wedge T(\operatorname{ATLEFT}(O, \text { Tar })) \Rightarrow T(D A L(O, \text { Tar })) \\
& T(\text { FLRS }(O)) \wedge T(\text { FLRS }(\text { Tar })) \wedge T(\text { ATRIGHT }(O, \text { Tar })) \Rightarrow T(\text { DAR }(O, \text { Tar })) \\
& T(\operatorname{FLRS}(O)) \wedge T(\operatorname{FLRS}(\text { Tar })) \wedge T(\operatorname{ATLEFT}(O, \text { Tar })) \Rightarrow F(D A R(O, \text { Tar })) \\
& T(\operatorname{FLRS}(O)) \wedge T(\operatorname{FLRS}(\text { Tar })) \wedge T(\operatorname{ATRIGHT}(\mathrm{O}, \mathrm{Tar})) \Rightarrow F(\operatorname{DAL}(\mathrm{O}, \mathrm{Tar})) \\
& T(\operatorname{FLRS}(O)) \wedge T(\operatorname{FLRS}(\text { Tar })) \Rightarrow F(D O T(O, T a r)) \\
& T(\operatorname{FLRS}(O)) \wedge T(\text { FLRS }(\text { Tar })) \Rightarrow F(D U N(O, \text { Tar })) \\
& T(\operatorname{FLRS}(O)) \wedge T(\operatorname{FLRS}(\text { Tar })) \Rightarrow F(\operatorname{DIF}(O, \text { Tar })) \\
& T(F L R S(O)) \wedge T(F L R S(\text { Tar })) \Rightarrow F(D A B(O, T a r)) \\
& T(\operatorname{FTB}(O)) \wedge T(F T B(\text { Tar })) \wedge T(\operatorname{HIGHER}(O, \text { Tar })) \Rightarrow T(\text { DOT }(\text { O,Tar })) \\
& T(F T B(O)) \wedge T(F T B(\text { Tar })) \wedge T(\operatorname{HIGHER}(O, \operatorname{Tar})) \Rightarrow F(D U N(O, T a r)) \\
& T(F T B(O)) \wedge T(F T B(\text { Tar })) \wedge T(\operatorname{LOWER}(O, T a r)) \Rightarrow F(D O T(O, T a r)) \\
& T(F T B(O)) \wedge T(F T B(\text { Tar })) \wedge T(\operatorname{LOWER}(O, \text { Tar })) \Rightarrow T(D U N(O, T a r)) \\
& T(F T B(O)) \wedge T(F T B(\text { Tar })) \Rightarrow F(D I F(O, \text { Tar })) \\
& T(F T B(O)) \wedge T(F T B(\text { Tar })) \Rightarrow F(D A B(O, \text { Tar })) \\
& T(F T B(O)) \wedge T(F T B(\text { Tar })) \Rightarrow F(D A L(O, \text { Tar })) \\
& T(F T B(O)) \wedge T(F T B(\text { Tar })) \Rightarrow F(D A R(O, \text { Tar })) \\
& T(F F B S(O)) \wedge T(F F B S(\text { Tar })) \wedge T(\operatorname{INFRONT}(O, T)(O, \text { Tar })) \\
& \Rightarrow T(D I F(O, T a r)) \\
& T(F F B S(O)) \wedge T(F F B S(\text { Tar })) \wedge T(B E H I N D(O, T)(O, \text { Tar })) \\
& \Rightarrow T(D A B(O, \text { Tar }))
\end{aligned}
$$




$$
\begin{aligned}
& T(\text { FFBS }(O)) \wedge T(\text { FFBS }(\text { Tar })) \wedge T(\operatorname{INFRONT}(O, T)(O, \text { Tar })) \\
& \Rightarrow F(D A B(O, \text { Tar })) \\
& T(F F B S(O)) \wedge T(F F B S(\text { Tar })) \wedge T(B E H I N D(O, T)(O, \text { Tar })) \\
& \Rightarrow F(D I F(O, \text { Tar }))
\end{aligned}
$$

